\title{
Studies on the Synthesis of Perfluoroaryl Sulfides and their Application in Desulfurative Nickel-Catalyzed Reductive Cross- Coupling
}

Shao-Chi Lee ${ }^{1}$, Che-Ming Hsu ${ }^{1}$, Hsin Kao ${ }^{1}$, Li-Yun Li, Zong-Nan Tsai, Yong-Ting Tsao, Yu-Ling Hsu, Shinje Miñoza, Cheng-chau Chiu* and Hsuan-Hung Liao ${ }^{2, * *}$

Department of Chemistry, National Sun Yat-sen University, Kaohsiung 80424, Taiwan

${ }^{1}$ These authors contributed equally, ${ }^{2}$ Lead contact

*Correspondence: ccchiu@mail.nsysu.edu.tw

**Correspondence: hsuan-hung.liao@mail.nsysu.edu.tw

\section{INTRODUCTION}

Nucleophilic aromatic substitution $\left(\mathrm{S}_{N} \mathrm{Ar}\right)$ reactions are widely applied in chemical research to build up complex molecular structures in aromatic systems ${ }^{1-3}$ An investigation reported by Brown and Boström confirmed that $S_{N} A r$ is the second most frequently occurring reaction in modern medicinal chemistry. ${ }^{4-6}$ Conventionally, the nucleophilic attack/ nucleofuge loss on an electron-poor $\pi$ system is described as a two-step mechanism, proceeding via a non-aromatic Meisenheimer complex. ${ }^{1}$ However, in recent years, it has been reported that some $S_{N} A r$ reactions, particularly those involving electron-rich arenes, operate through a concerted pathway rather than a stepwise one. This finding not only revolutionized the understanding of the mechanistic aspects of these classical reactions but also suggested that, using $S_{N} A r$ chemistry, electron-rich arenes might be converted into a much wider range of products than previously anticipated. ${ }^{7-15}$

Fluorinated arenes, on the other hand, have recently gained prominence in the areas of materials science and pharmaceutical chemistry owing to their distinct chemical and physiological properties, e.g., enhanced metabolic stability and improved lipophilicity. ${ }^{16-22}$ However, there is no known natural source for fluoroarenes which means that these compounds must be prepared synthetically. The sequential introduction of multiple fluorine atoms into an aromatic ring is difficult as such processes usually require the aromatic ring toeature very specific functional groups as indicated in Scheme $1 \mathrm{~A}(\mathrm{a})$. This is further complicated by the poor availability of F-reagents. ${ }^{23-25}$ Currently, the predominant strategy to synthesize polyfluorinated arenes relies on the "reverse" reaction, namely, to start from readily available perfluoroarenes and selectively convert one or more fluoro groups to other functional groups, as illustrated in Scheme $1 \mathrm{~A}(\mathrm{~b}){ }^{26-29}$ Over the past years, significant efforts have been devoted to the development of transition-metal catalysis to selectively activate strong covalent $\mathrm{C}_{\text {aryl }}-\mathrm{F}$ bonds ${ }^{30-32}$ as well as the photocatalytic fragmentation of perfluoroaryl fluorides. ${ }^{33-36}$ However, both strategies still feature crucial limitations: for instance, to activate a $\mathrm{C}_{\text {aryl }}-\mathrm{F}$ bond with a metal catalyst, it is required to have a directing group in the ortho-position. In addition, the use of elaborate (photo-)catalysts not only increases the cost of the process but may also result in residual metal impurities in the final products. ${ }^{35-}$

${ }^{36}$ Under the aspect of practicality, the ideal transformation would be transition metal-free that allows the rapid synthesis of versatile polyfluoroarenes with excellent regioselectivity.

In particular, the development of methods to introduce sulfur selectively into polyfluoroarenes is of growing importance, as polyfluoroaromatic sulfur-containing compounds are often encountered in 
pharmaceuticals (Scheme 1B), agrochemicals, and functional materials. ${ }^{37-40}$ While recent developments have made polyfluoroaryl-sulfides synthetically accessible, ${ }^{41-47}$ some reported synthetic processes still suffer from limitations, including poor regioselectivity. ${ }^{41-44}$ Other strategies are rather complex and require the preparation of hetarenium salts for the synthesis. ${ }^{43-44}$ There are also other methods that have only been used in very focused settings, such as the preparation of polymers ${ }^{45}$ and peptide stapling. ${ }^{46-47}$ To deal with these issues, we attempted to develop a metal-free defluorosulfurization method for the synthesis of perfluoroarenes using $S_{N} A r$ chemistry, with a focus on the following considerations: 1) highly selective substitution of $\mathrm{C}-\mathrm{F}$ bonds by mercaptans or thiolates; and 2) broad applicability with high functional group tolerance, making it suitable for the modification of complex molecules. In this report, we investigate the $S_{N} A r$ reactions of a broad spectrum of perfluoroarenes with mercaptans of varying electronic properties. This work further makes use of quantum-chemical simulations to rationalize the bond-forming/cleaving mechanism in the $\mathrm{S}_{N} \mathrm{Ar}$ step. Additionally, we demonstrate how organic synthesis can exploit the perfluoroarylsulfides formed in the process described above by embarking upon the study of nickel catalyzed reductive Liebeskind-Srogl-type arylations, wherein the perfluoroaryl attached to the $S$ atom acts as a redox-active scaffold to activate the $\mathrm{C}-\mathrm{S}$ bond in the initial mercaptan. ${ }^{48-52}$

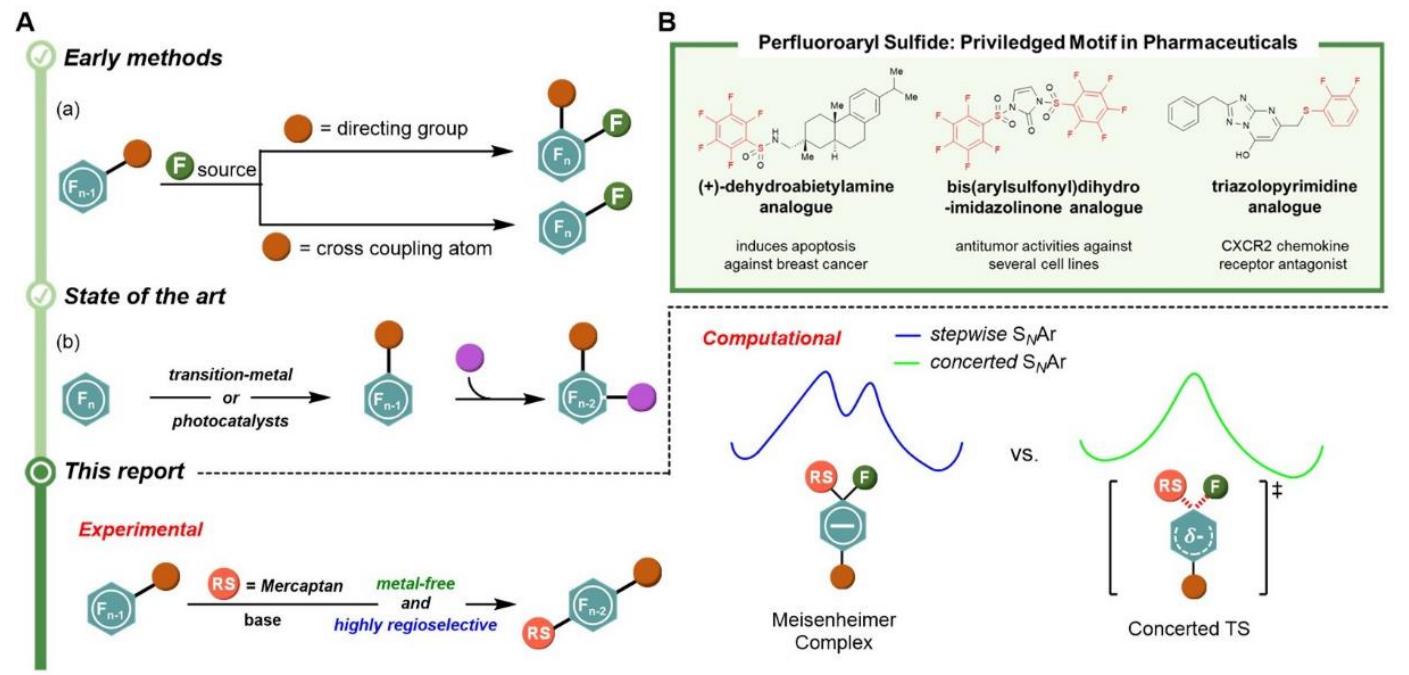

Scheme 1. (A) Literature strategies to synthesize polyfluoroarenes compared to the reaction studied in the present work. (B) Representative examples of sulfur-bound poly- and per-fluoroarenes used as pharmaceutical agents.

The activation of $\mathrm{C}-\mathrm{S}$ bonds is a challenging yet important topic in chemical processing. Apart from applications in chemical synthesis, it also plays an important role in the context of sulfur removal in petrochemical processes, as residual sulfur can lead to the formation of acid rain and other pollutantrelated issues. ${ }^{53}$ Therefore, the development of new $\mathrm{C}-\mathrm{S}$ activation approaches is of considerable interest for the desulfurization process in petrochemical processes. ${ }^{53-54} \mathrm{It}$ would be desirable to have a strategy that does not require stoichiometric amounts of highly sensitive and expensive organometallic reagents, as needed for the conventional Liebeskind-Srogl cross-coupling. In this article, we attempt to achieve this via a reductive cross-coupling step, a strategy that activates the $\mathrm{C}-\mathrm{S}$ bond to obtain an electrophile, which is then coupled to a second electrophile, replacing the original $\mathrm{C}-\mathrm{S}$ bond with a $\mathrm{C}-\mathrm{C}$ bond. ${ }^{55-57}$ One of the early concepts for $\mathrm{C}-\mathrm{S}$ bond activation is reductive desulfurization, first reported by 
Bougault in 1940, which uses a stoichiometric amount of Raney nickel as a single electron donor. ${ }^{58-59}$ This hydrodesulfurization process is proposed to occur via fission of the $\mathrm{C}-\mathrm{S}$ bond to give radical intermediates, followed by a hydrogen atom transfer to forge a new $\mathrm{C}-\mathrm{H}$ bond. However, that process only allowed the removal of mercapto and other sulfur-containing groups. The concept of $\mathrm{C}-\mathrm{S}$ bond activation has also been exploited for the functionalization of $\mathrm{C}-\mathrm{H} / \mathrm{C}-\mathrm{X}$ bonds ( $\mathrm{X}=$ halide). Organosulfur reagents like thianthrene sulfoxide, ${ }^{60-63}$ dibenzothiophene $S$-oxide, ${ }^{64-65}$ xanthate ${ }^{66-67}$, thiocarbamate, ${ }^{68-69}$ and tetrafluoropyridinyl disulfide ${ }^{48-52}$ can be added to arenes or organohalides to obtain sulfonium salts or sulfides, which contain a weak $\mathrm{C}-\mathrm{S}$ bond that can be easily replaced in a reductive $\mathrm{C}-\mathrm{S}$ bond cleavage step. Despite the success of the above methods, which are all based on C-S bond activation, one still lacks a generally applicable tool for the efficient conversion of mercapto groups into other functionalities. Recently, some elegant synthetic applications of alkyl radicals obtained through the desulfurization of mercaptans have been reported. Following their generation, the radicals can be added to unsaturated systems to access products of a formal alkylation process. ${ }^{70-72}$ However, to the best of our knowledge, the application of alkyl radicals, generated in this manner, for arylation chemistry has not yet been reported. Nevertheless, we hypothesize that the alkyl radical could be intercepted by an aryl nickel (II) species to form a reactive $\mathrm{Ni}^{\text {III intermediate, }}{ }^{73-76}$ that gives rise to a desulfurized arylation product after a facile reductive elimination (Scheme 2). In this study, we confirm our hypothesis and describe the first example of nickel-catalyzed reductive desulfurization leading to a direct $C\left(s p^{2}\right)-C\left(s p^{3}\right)$ cross-coupling. ${ }^{77}$

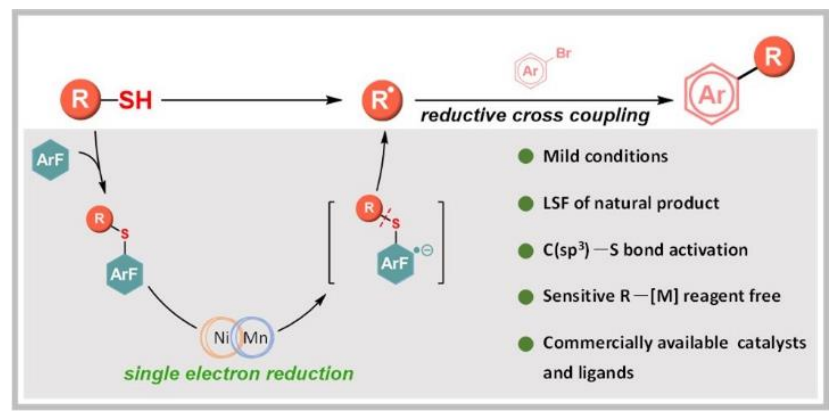

Scheme 2. Nickel-catalyzed desulfurative cross-coupling of activated thioether with aryl halide RESULTS AND DISCUSSION

\section{Defluorosulfurization on Perfluoroarenes - Optimization}

We started our investigation by selecting pentafluoropyridine 1a as a representative perfluoroarene and thiol $\mathbf{2} \mathbf{a}$ as model substrates to examine the viability of our metal-free defluorosulfurization strategy (Table 1). After a thorough examination of the reaction conditions, we were delighted to find that employing triethylamine $(0.3 \mathrm{mmol})$ in acetonitrile afforded 3a as a single regioisomer in $99 \%$ GC yield (Entry 1). The same reaction was performed on a gram-scale and 3a was isolated in $95 \%$ yield, demonstrating excellent amenity towards scale-up. In the screening of other bases, pyridine (15\% yield, Entry 2 ) and 2,6-lutidine ( $52 \%$ yield, Entry 3 ) were found to be less effective, which we attributed to their lower basicity compared to triethylamine. We also tested $\mathrm{Na}_{2} \mathrm{CO}_{3}$ in $\mathrm{DMSO}^{78}$ as an inorganic base; however, an inferior result was obtained (73\% yield, Entry 4$)$. On the other hand, changing acetonitrile to toluene only led to recovery of the starting materials (Entry 5). Employing DMSO, however, also gave a commendable yield ( $97 \%$ yield, Entry 6 ). 
Table 1. Optimization of the reaction conditions

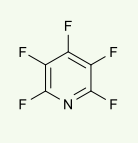

1a

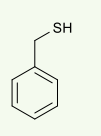

2a

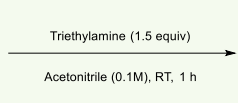

Acetonitrile $(0.1 \mathrm{M}), \mathrm{RT}, 1 \mathrm{~h}$

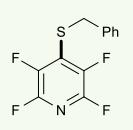

3a

\begin{tabular}{lll}
\hline Entry & Variation from optimized condition & Yield $^{\mathrm{b}}$ \\
\hline 1 & none & $99(95)^{c}$ \\
\hline 2 & Pyridine instead of Triethylamine & 15 \\
\hline 3 & 2,6 -lutidine instead of Triethylamine & 52 \\
\hline $4^{d}$ & $\mathrm{Na}_{2} \mathrm{CO}_{3}$ & 73 \\
\hline 5 & Toluene instead of Acetonitrile & \\
\hline 6 & DMSO instead of Acetonitrile & \\
\hline & & \\
\hline 7 & Reaction for 30min & 97 \\
\hline 8 & 2 mmol Triethylamine & 90 \\
\hline 9 & Without Triethylamine & N.R. ${ }^{\mathrm{f}}$
\end{tabular}

aReaction conditions: $1 \mathrm{a}(0.2 \mathrm{mmol}), 2 \mathrm{a}(0.2 \mathrm{mmol})$, trimethylamine $(0.3 \mathrm{mmol})$, acetonitrile $(2.0 \mathrm{~mL})$, room temperature, $1 \mathrm{~h}$.

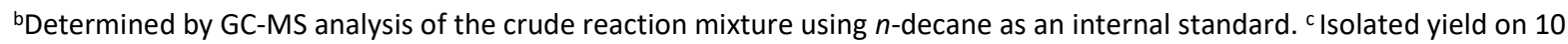
mmol scale after purification by column chromatography. ${ }^{d}$ DMSO as solvent. eOnly starting material observed. fStarting material and disulfude product observed. See the Supporting Information for more entries in our optimization studies.

Nonetheless, we opt to use acetonitrile as solvent, which is recommended as a green polar aprotic reaction medium. ${ }^{79}$ Reducing the reaction time to 30 mins ( $84 \%$ yield, Entry 7 ) and the triethylamine amount to $2 \mathrm{mmol}$ ( $90 \%$ yield, Entry 8) also resulted in lower yields. In the absence of triethylamine, no desired product was detected, which shows the essential role of a base in this $S_{N} A r$ reaction (Entry 9). It is also noteworthy that no other chemical entities such as pentafluoropyridine-based hetarenium salts from the para-substitution of the pentafluoroarene with triethylamine, or products from dimerization/oligomerization pathways were detected during our optimization studies. We further denied the possibility of having a hetarenium intermediate, which could act as an activated substrate possessing a good leaving group, and initially hypothesized that our strategy proceeds via direct substitution of thiol 2a. ${ }^{43-44}$ Moreover, a DFT calculation was carried out, and we validated that hetarenium intermediate formation is impeded by its higher energy barrier $(173.24 \mathrm{~kJ} / \mathrm{mol})$ than that for the thiolation pathway $(67.96 \mathrm{~kJ} / \mathrm{mol}$, see section 9.1 in the Supplemental Information). A more detailed discussion on the thiolation pathway will be provided later in context of the discussion on Figure 1.

\section{Defluorosulfurization on Perfluoroarenes - Substrate scope}

With the optimal condition in hand, the scope of substituents for our defluorosulfurization reaction was thoroughly investigated (Scheme 3). Pleasingly, excellent yields and only a single regioisomer were observed in all cases. We first conducted defluorosulfurization in the presence of electron-rich $p$-methyl (2b) and $p$-methoxy (2c) benzyl mercaptans to compare their reactivity with the electronically neutral benzyl mercaptan 2a; and gratefully, excellent yields were obtained. In the same way, a series of electron withdrawing-substituted thiol substrates were examined, such as halogen-substituted benzylic mercaptans, which provided outstanding yields (3d-3e) and offer an opportunity for further 


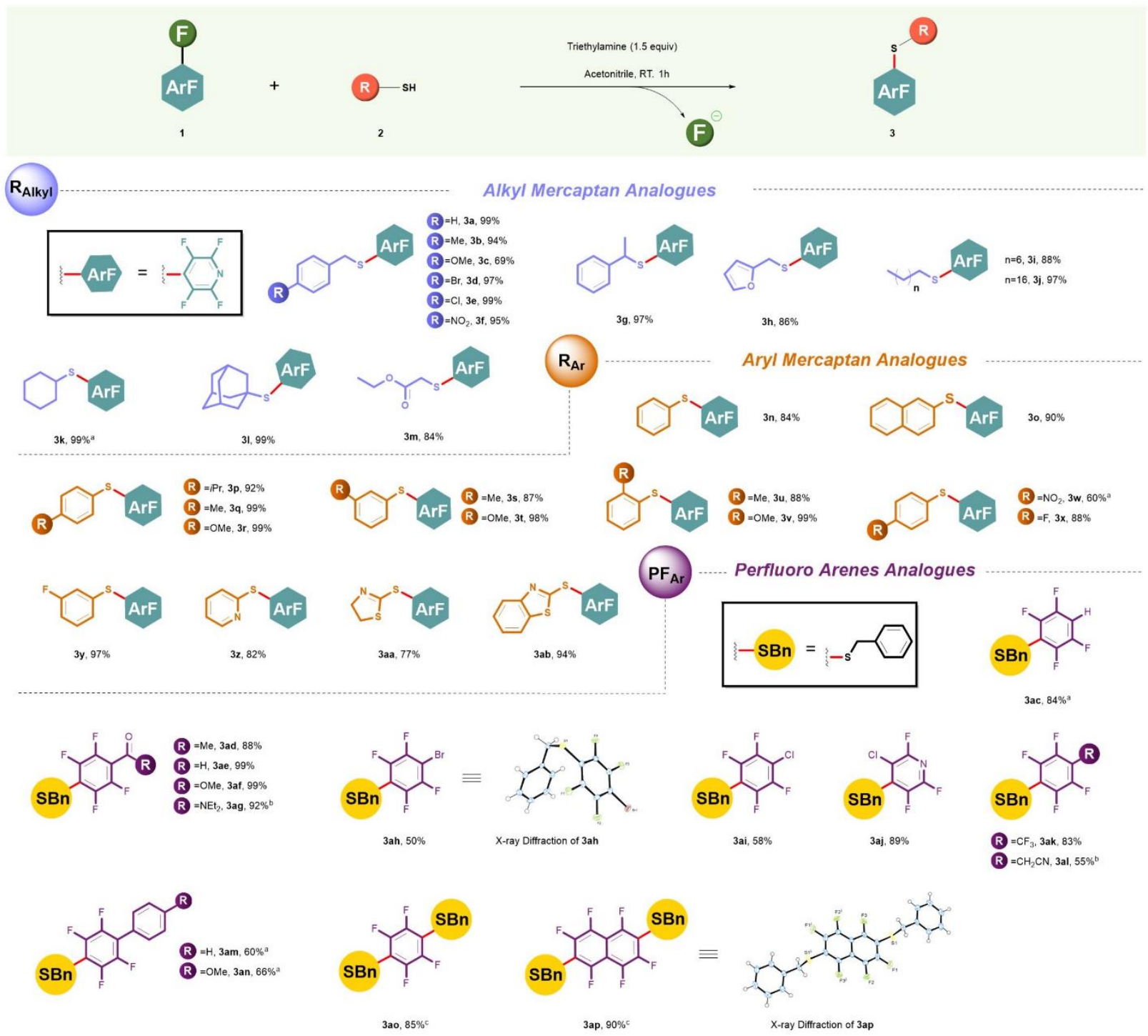

Scheme 3. Scope of $S_{N} A r$ defluorosulfurization of mercaptans and perfluoroarenes

Reaction conditions: $1(0.2 \mathrm{mmol}), 2(0.2 \mathrm{mmol})$, triethylamine $(0.3 \mathrm{mmol})$, acetonitrile $(2.0 \mathrm{~mL}), \mathrm{N}_{2}, 1 \mathrm{~h}$. a Reaction performed for $3 \mathrm{~h}$. b2 $(0.3 \mathrm{mmol})$, triethylamine $(0.5 \mathrm{mmol})$. c $2(0.4 \mathrm{mmol})$, triethylamine $(0.5 \mathrm{mmol})$. See Supporting Information for full experimental details.

derivatization. Additionally, a nitro group was well tolerated and gave the corresponding perfluorothiol $\mathbf{3 f}$ in $\mathbf{9 5 \%}$ yield. A secondary mercaptan was also evaluated and delivered product $\mathbf{3 g}$ in similarly high yield. This result is particularly valuable as the secondary benzylic sulfide motif is often found in therapeutic agents like montelukast. ${ }^{80}$ We further modified the aromatic system from benzene to furan (2h), and a good yield was given. Aliphatic systems, such as primary alkyl mercaptans with differing chain length (2i-2j) and secondary cycloalkyl mercaptans (2k), were also found to afford $\mathbf{3} \mathbf{i}-\mathbf{3 k}$ in good yields. Hence, we could conclude that neither electron density nor aromaticity itself is crucial for the reactivity of the benzylic mercaptans. Our Hammett plot depicted in the SI (Section 8.1.1) also 
corroborates this observation. In contrast to other substrates, bulky adamantyl-containing mercaptan 2I, which imposes steric hindrance, was found to be congruous to our developed protocol and gave a quantitative yield. Finally, mercaptan $\mathbf{2 m}$ containing an electron-deficient ester, which could be further functionalized, was also found to be tolerated under our reaction conditions.
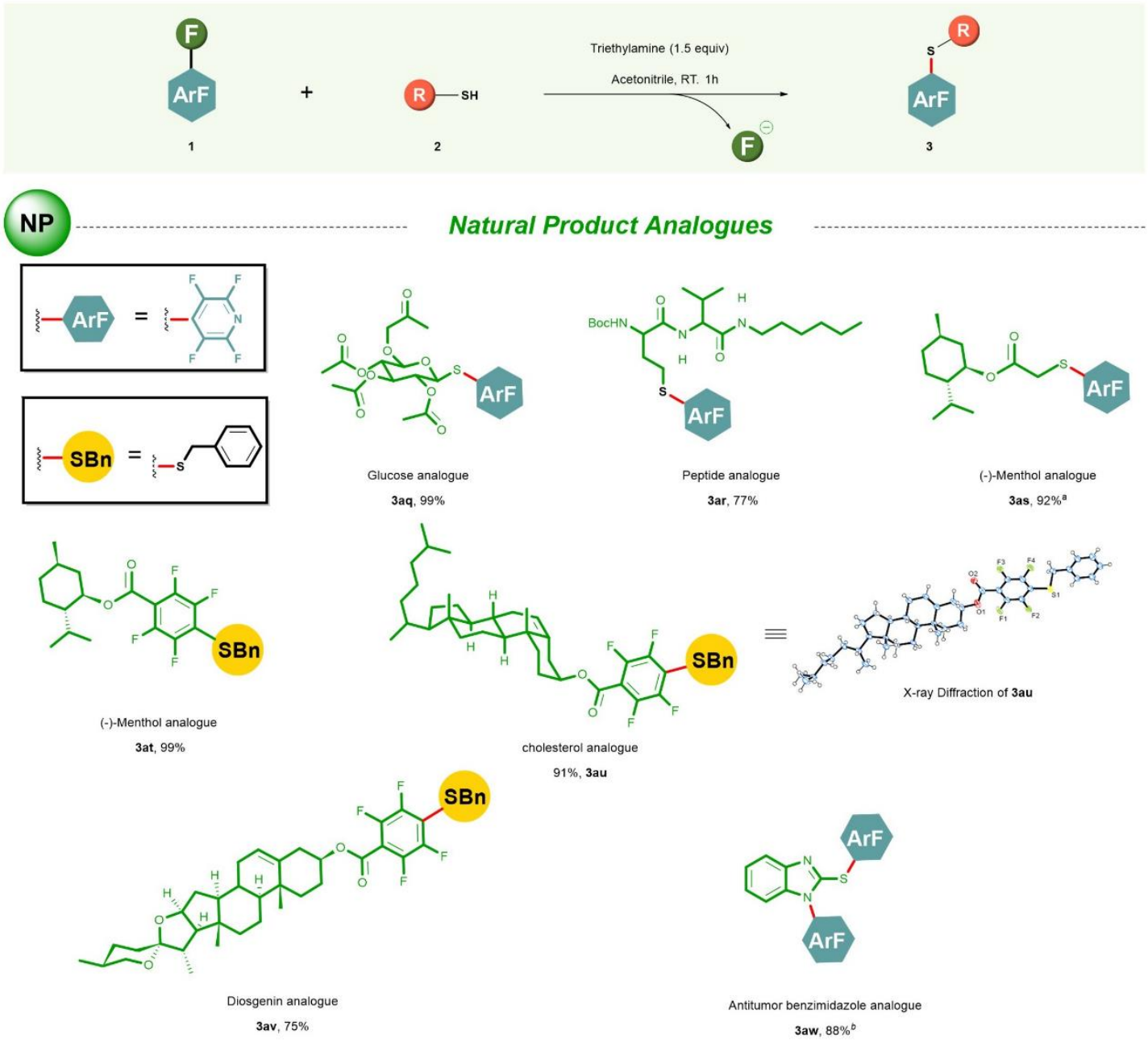

\section{Scheme 4. Late-stage application of $S_{N} A r$ defluorosulfurization}

Reaction conditions: $1(0.2 \mathrm{mmol}), 2(0.2 \mathrm{mmol})$, triethylamine $(0.3 \mathrm{mmol})$, acetonitrile $(2 \mathrm{~mL}), 1 \mathrm{~h}$. aPerformed in $10 \mathrm{mmol}$ scale. ${ }^{\mathrm{b}} 1(0.5 \mathrm{mmol}), 2(0.2 \mathrm{mmol})$, triethylamine $(0.7 \mathrm{mmol})$, acetonitrile $(2 \mathrm{~mL}), 12 \mathrm{~h}$. See the Supporting Information for full experimental details.

After demonstrating functional group tolerance of alkyl and benzyl mercaptans, we were keen to investigate aryl mercaptans as nucleophiles for defluorosulfurization. Compared to unsubstituted phenyl $\mathbf{2 n}$ and naphthyl $\mathbf{2 0}$ mercaptans, electron-donating substituents were beneficial to the nucleophilicity of mercaptans and further improved the yield regardless of the position: para (2p-2r), meta (2s-2t), or ortho (2u-2v). On the other hand, an electron-withdrawing substituent, such as nitro-containing mercaptan $\mathbf{2 w}$, provided the corresponding product in a relatively low yield. As anticipated, these results are consonant to substituent effects. Interestingly, the yields for reactions with fluoridecontaining mercaptans were found to be superior to thiophenol $\mathbf{2} \mathbf{n}$ at both para (2x) and meta (2y) positions. The compatibility of pyridine- (2z), thiazoline- (2aa), and benzothiazoline-containing 
mercaptans (2ab) were also tested, demonstrating tolerance towards heterocyclic scaffolds. We were pleased to find that all of them delivered the expected products in high yields. In summary, various mercaptans not only underwent our defluorosulfurization reaction in high yields but also presented absolute preference to the para-regioisomer. We further performed a simple computational inquiry on the perceived regioselectivity of our $S_{N} A r$ reaction and found out that substitution at the para-position is energetically favorable compared to the ortho-site. (See SI section 9.3)

To further demonstrate the flexibility of this methodology, we turned our attention to the scope of the perfluoroarene reaction partner. The chemoselectivity of our protocol was further tested by probing a series of para-substituted pentafluorobenzene. Nevertheless, electron-withdrawing groups such as unsubstituted pentafluorobenzene (1ac), ketone (1ad), aldehyde (1ae), ester (1af), and tertiary amide (1ag) were all found to perform well under our reaction conditions. In addition, para-substituted bromoand chloro-substituted pentafluorobenzenes (1ah and 1ai-aj, respectively) were also tolerated, albeit in lower yields. The perceived compatibility with halides is due to the fact that substitution of fluoride is more kinetically favorable than bromide and chloride. Density Functional Theory (DFT) calculations also confirmed that the latter two have energy barriers that are at least $30 \mathrm{~kJ} / \mathrm{mol}$ higher (SI section 9.2). This tolerance towards halide substituents provides the opportunity for further derivatization, for example, with traditional cross-coupling chemistry or photocatalysis. ${ }^{81}$ Additionally, perfluoroarenes bearing electron-withdrawing trifluoromethyl and cyanomethyl groups (1ak-1al) performed well, as did $\pi$ electron extended analogues such as phenyl (1am) and methoxyphenyl (1an) derivatives. Moreover, the scope of this defluorosulfurization could be broadened to disubstituted species by fine-tuning the reaction stoichiometry. Hexafluorobenzene (1ao) and octafluoronaphthalene (1ap) could react with two mercaptan groups and give a potential fixed and planar linker (3ao-3ap), ${ }^{45-47}$ which could be furthermore applied to bioconjugation and materials chemistry.

Prompted by the excellent functional group tolerance of the methodology, the scope was further expanded to more complex molecules. We successfully embellished both nucleophilic and electrophilic building blocks with high chemoselectivity for further late-stage functionalization (Scheme 4). A glucose derivative, modified with a secondary mercaptan, participated in the $S_{N} A r$ reaction with perfluoroarene $1 \mathrm{a}$ and afforded 3aq in excellent yield. Other natural product analogues like peptide 2ar and (-)-menthol derivative 2 as proved to be competent nucleophiles and delivered the corresponding products 3ar-3as in high yield. Similar success was observed with the evaluated electrophiles. All elaborate pentafluoropyridine compounds, including (-)-menthol (1at), cholesterol (1au), and diosgenin (1av) derivatives have been transformed into the corresponding products in high yields. The power of this methodology has also been applied to the synthesis of a dipentafluoropyridinylated anticancer compound 3aw in a gratifying yield. ${ }^{84}$ To summarize, this simple defluorosulfurization strategy was compatible with various natural products and pharmaceutical derivatives. 


\section{Defluorosulfurization on Perfluoroarenes - Mechanistic investigation}

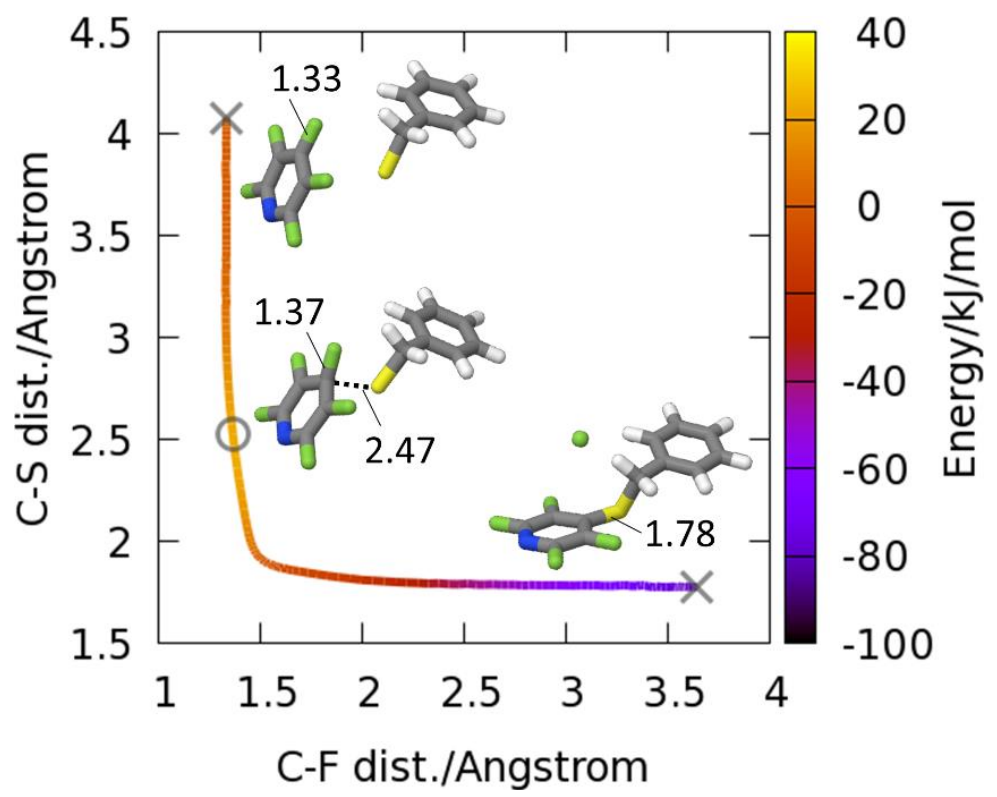

Figure 1. Reaction energy profile for the $S_{N} A r$ reaction of pentafluoropyridine 1 a in acetonitrile computed at B3LYP level.

An $\mathrm{F}^{-}$of $1 \mathrm{a}$ is being substituted by $\mathrm{Ph}-\mathrm{CH}_{2}-\mathrm{S}^{-} 2 \mathrm{a}^{-}$. The molecular structures are the optimized TS (0) as well as the IS and product (both $\mathrm{x}$ ) as obtained from the IRC analysis. The energy of the IS, pentafluoropyridine and $\mathrm{Ph}-\mathrm{CH}^{-}-\mathrm{S}^{-}$is arbitrarily set to 0 . The color scheme represents the electronic energy without zero-point energy (ZPE) correction.

After having dealt with the application of the $S_{N} A r$ reaction, we now turn to look at the mechanistic details. Earlier studies by the group of Jacobsen have introduced a Marcus-type picture to discuss the $\mathrm{S}_{N} \mathrm{Ar}$ mechanism. ${ }^{83}$ They found that the relative stability of the potential energy surfaces (PESs) associated with the initial state (IS), the Meisenheimer-type structure (MTS), as well as the product, determines whether the MTS represents a minimum (commonly referred to as "Meisenheimer complex") or a transition state (TS). In other words, this decides whether a $S_{N} A r$ pathway proceeds via a concerted or a stepwise mechanism. As the MTS features both the nucleophile and the leaving group attached to the aromatic ring, it typically is a negatively charged structure. This, in return, means that the corresponding PES is expected to shift down in energy, i.e., become more stable, if the aromatic ring is substituted with electron-withdrawing groups. As visualized in Figure 2D, a low-lying PES for the MTS is typically associated with a stepwise mechanism featuring the Meisenheimer complex as an intermediate, while a high lying PES leads to a concerted mechanism (Figure 2C). This idea is also reflected in a recent report by the groups of Murphy and Tuttle. ${ }^{84}$ Their study reported that the electron affinity of the aromatic substrate could be used as a simple descriptor to predict whether a specific $\mathrm{S}_{N} \mathrm{Ar}$ reaction will proceed via a stepwise or a concerted mechanism.

To shed light on the mechanisms of the defluorosulfurization $\mathrm{S}_{N} \mathrm{Ar}$ reaction considered in this work, a Hammett plot was constructed by considering the reactions of three different para-substituted polyfluoroarenes $\left(\mathrm{C}_{6} \mathrm{~F}_{5}-\mathrm{R}\right.$, with $\mathrm{R}=\mathrm{H}, \mathrm{Ph}$, and $\mathrm{CH}_{2} \mathrm{CN}$, see $\mathrm{SI}$, Section 8.1). Instead of obtaining a linear correlation, the plot resembled a $\mathrm{v}$-shaped curve. It has been previously established that a curved 
Hammett plot might suggest that not all reactions are following the same mechanism. ${ }^{85-86}$ Murphy and Tuttle also noted in their work that a Hammett plot might not be a suitable method for probing the mechanistic details of some $S_{N} A r$ reactions. ${ }^{84}$ Since the Hammett plot leaves some open questions, we have followed the idea of Murphy and Tuttle to use the electron affinity to find out more information about the reaction mechanism. We used DFT calculations to determine the electron affinities for 12 different perfluoroaryl substrates that were utilized in the experiments discussed as mentioned previously. However, nine out of the twelve examples feature electron affinities that are located in the "boundary region," where it is not possible to make a clear statement whether a $\mathrm{S}_{N} A r$ reaction follows a stepwise or concerted mechanism.

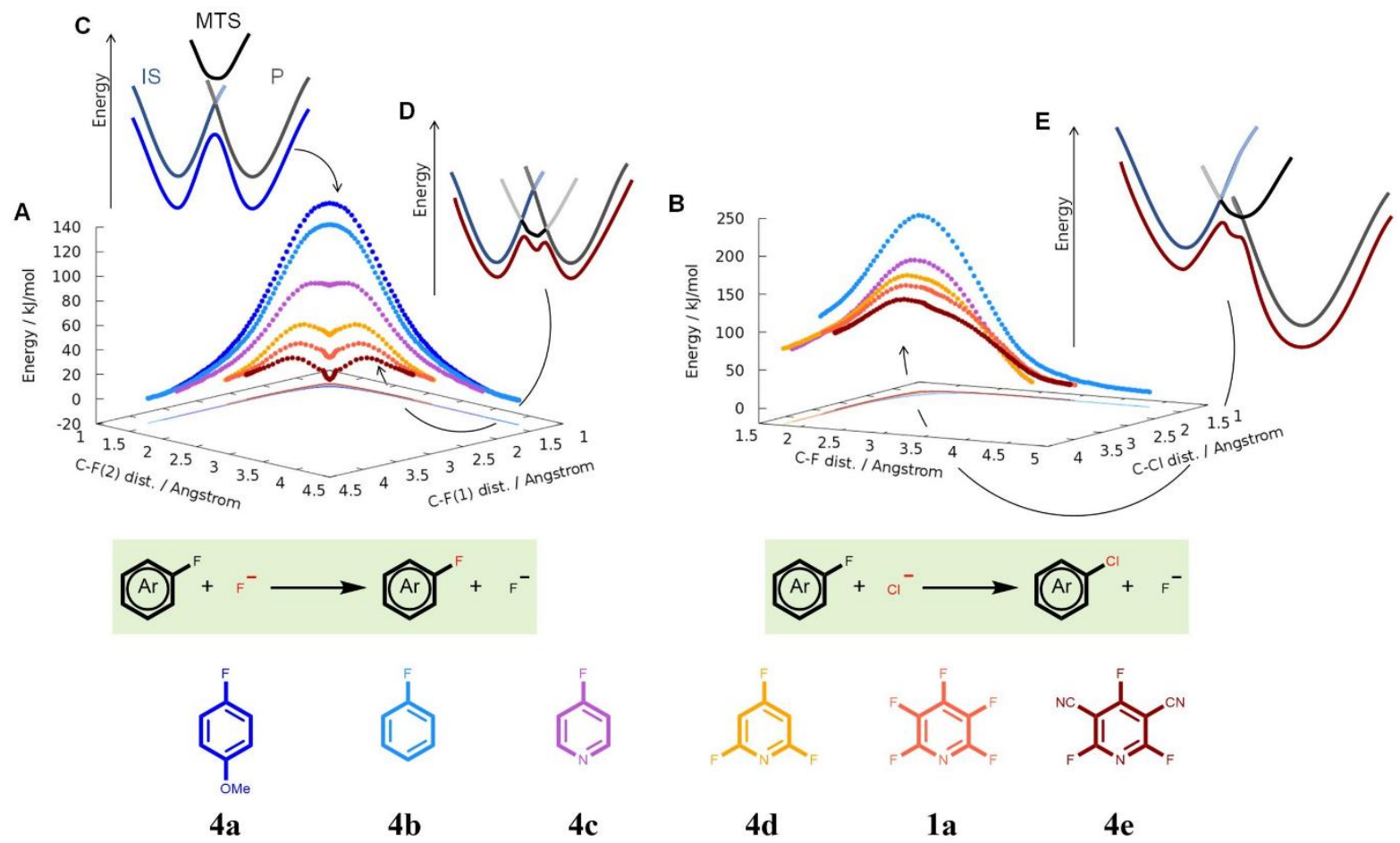

Figure 2. The reaction energy profiles as obtained from an IRC analysis for the $S_{N} A r$ reaction of fluorine-substituted aromatics.

The different colors are associated with the aromatic structure shown at the bottom of the figure. The $\mathrm{F}^{-}$in para-position to the pyridine-N/to the methoxy group is substituted by (A) another $\mathrm{F}^{-}$and $(\mathrm{B})$ a $\mathrm{Cl}^{-}-$ion, see the reactions in the green boxes. Figures (C), (D), and (E) provide a schematic representation of the Marcus-type pictures that can be used to rationalize the shape of the energy profiles. Note the small shoulder in the plot for the $\mathrm{Cl}$ - substitution of the electron poorest system 2,4-Dicyano-1,3,5trifluoro-pyridine in (B). For this system, the IRC analysis stops at this "flat" area of the reaction pathway. The shown reaction pathway contains the data from two separated calculations.

The calculated data here are supplied in the SI, Section 9.4. It seems that there is no easy way to determine the reaction mechanism for the studied reactions. Thus, we have decided to explore reactions pathways computationally by explicitly searching for the TSs and intermediates of the reactions. For our prototypical reaction, the defluorosulfurization of pentafluoropyridine 1a with benzylthiolate (here abbreviated as $\mathbf{2} a$; , the deprotonated form of $\mathbf{2 a}$ ), the calculated reaction energy profile is displayed in Figure 1. As visible, only one TS structure has been identified. In the TS structure, 
both the C-F and the C-S distances are, with values of $1.37 \AA$ and $2.47 \AA$, slightly longer than the equilibrium distances found in the IS and the product. The reaction pathway obtained from the IRC analysis in Figure 1 confirms that this $S_{N} A r$ reaction follows a concerted mechanism. In other words, there is only one TS and no Meisenheimer complex intermediate.

Figure 1 provides some additional information about the mechanism of this $S_{N} A r$ reaction. Although the reaction follows a concerted mechanism, the $\mathrm{C}-\mathrm{F}$ bond cleavage and the $\mathrm{C}-\mathrm{S}$ bond formation are somewhat asynchronous. The reaction starts with the approach of the $\mathrm{Ph}-\mathrm{S}^{-}$nucleophile, while the $\mathrm{C}-\mathrm{F}$ bond length is only weakly affected. Even at the TS, the C-F bond is only $3 \%$ longer than the equilibrium bond length. The elongation of the $\mathrm{C}-\mathrm{F}$ essentially occurs after the $\mathrm{C}-\mathrm{S}$ bond has been formed, which can be deduced from the bent shape of the graph. This means that the reaction features an early TS, as expected for the strongly exothermic reaction, with a Gibbs free energy of reaction of $\Delta G_{r}=-68.1$ $\mathrm{kJ} / \mathrm{mol}$. Following the concepts of earlier studies, ${ }^{83-84}$ we were surprised by the computed mechanism for this reaction. The calculated electron affinity for $1 \mathrm{a}$ is $-39 \mathrm{~kJ} / \mathrm{mol}$. This value falls in a range where a simple prediction of the reaction mechanism is not possible, according to Murphy and Tuttle. ${ }^{84}$ However, we have expected that the fluoro groups of the perfluorinated arene should be sufficiently electronwithdrawing to yield a system that proceeds via the formation of a Meisenheimer complex.

To rationalize this unexpected result, we decided to extend the Marcus-type picture used by Jacobsen and colleagues and combine it with the Brønsted-Evans-Polanyi (BEP) principle that is often encountered in the context of heterogeneous catalysis. ${ }^{87}$ The BEP principle states that, for similar reactions, there is a linear dependency between the activation barrier and the reaction energy; the more exothermic a reaction is, the smaller the associated activation barrier. As the reaction of $\mathbf{1 a}$ and $2 a^{-}$is exothermic $(-68.1 \mathrm{~kJ} / \mathrm{mol})$, it is safe to assume that the associated MTS is significantly less stable than the product species. The lack of a TS structure connecting the MTS and the product species can be interpreted in the following way: Reaction from the MTS to the product is exothermic to the extent that the activation barrier approaches zero. In this picture, the considered reaction can therefore be understood as a "stepwise" $S_{N} A r$ mechanism where the formation of the MTS from the IS is a reaction with a barrier, while the subsequent rearomatization of the MTS is barrierless. Describing the reaction this way has the advantage that it explains the results of Figure 1; the elongation of the C-F bond leading to the bond cleavage begins after the $\mathrm{C}-\mathrm{S}$ bond has been formed.

Alternatively, the presence of only one transition state in the reaction can also be rationalized using just the Marcus-type picture, similar to the situation shown in Figure $2 \mathrm{E}$. As the product is significantly more stable than the MTS, the potential energy surface of the former is energetically low-lying. If the energy difference between the MTS and the product state becomes sufficiently large, the potential energy surfaces of the two structures cross before the minima on the potential energy surface of the MTS is reached. In this case, one would only observe one TS along the reaction pathway. However, the potential energy surface of the MTS is also involved in the reaction, unlike the situation sketched in Figure $2 \mathrm{C}$. Note that the above model used to explain the lack of a second TS in the $\mathrm{S}_{N} \mathrm{Ar}$ process follows the same logic used to describe the "inverted region" in the context of the Marcus theory. ${ }^{88}$ To support the explanation above, we designed a set of model reactions. We considered various fluorinesubstituted benzene and pyridine derivatives and investigated the substitution of the fluoro group by a fluoride and by a chloride ion, respectively. The considered substrates reach from 1-fluoro-4methoxybenzene (4a) that contains electron-donating substituents, to 2,4,6-trifluoro-3,5dicyanopyridine (4e), which possesses several electron-withdrawing substituents. Note that the 
substitution by a fluoride ion, as illustrated in Figure $2 \mathrm{~A}$, can be understood as a thermo-neutral selfexchange reaction. At variance, the substitution reactions by a chloride ion in Figure $2 \mathrm{~B}$ represent exothermic processes with reaction energies derived from the IRC analysis between -50 and -100 $\mathrm{kJ} / \mathrm{mol}$. For the self-exchange reactions, one sees that the $\mathrm{S}_{N} \mathrm{Ar}$ processes at the electron-rich aromatics feature the MTS as a TS. As the aromatic substrates become less electron-rich, the MTS-PES shifts down in energy, so the MTS becomes a minima state yielding a stepwise $S_{N} A r$ mechanism. The chargestabilizing effect of the electron-withdrawing groups becomes so pronounced that the MTS of the 2,4,6trifluoro-3,5-dicyanopyridine (4e) system becomes even more stable than the reactant and product. When we consider the substitution of a fluoro group by a chloride ion, we see how the energy difference between the product and the initial states affects the energy profile of the reaction. For nearly all considered systems, except 2,4,6-trifluoro-3,5-dicyanopyridine (4e), one sees a reaction profile with only one TS. We interpret this as the situation illustrated in Figure $2 \mathrm{~B}$, where the PES of the MTS is too high in energy to play a role. For 2,4,6-trifluoro-3,5-dicyanopyridine (4e), a very electron-poor compound, the MTS is stable enough to be reflected as a small shoulder in the reaction energy profile close to the TS. As displayed in Figure 2E, the situation for this substrate is essentially the same as in the reaction of $\mathbf{1 a}$ with $\mathbf{2 a}$ - discussed earlier in this Article. To fully support our model explaining the mechanistic details of the investigated $S_{N} A r$ reactions, it would have been ideal to present a system with an even stronger electron deficiency. In this case, the substitution of the fluoro group by a chloride should become a stepwise process with two clear TSs. Thus, we also considered the substitution reaction of 4-fluoro-2,3,5,6-tetracyanopyridine. However, it seems that in this case, the potential energy surface of the MTS becomes so stable that no TS along the reaction pathway could be located. In other words, the entire $S_{N} A r$ reaction of that electron-poor compound can be thought of as a barrierless process.

\section{Defluorosulfurization on Perfluoroarenes - Applications}

Apart from investigating the substrate scope and the mechanistic details, we also wanted to make use of the defluorosulfurization and develop programmed selective $\mathrm{C}-\mathrm{F}$ bond activation strategies to access complex fluorinated aromatics with diverse functional groups. To transfer this concept into practice, we began with readily available perfluorinated arenes and subjected them sequentially to our $S_{N} A r$ reaction followed by transition metal-catalyzed and photocatalytic transformations.

The first example for our programmed selective $C-F$ bond activation strategies is a linear synthesis shown in Scheme 5a. It begins with a nickel-catalyzed cross-coupling between pentafluoropyridine 1a and phenylboronic acid under an aerobic atmosphere to afford the para-substituted product $\mathbf{5} .^{89} \mathbf{5}$ underwent hydrodefluorination under photocatalytic conditions to remove a fluorine atom at the meta position, yielding product $6 .^{81}$ Applying our $S_{N} A r$ method to replace the ortho fluorine group of 6 resulted in the thioalkylated product 7 in $85 \%$ yield. This result illustrates that in the absence of a suitable leaving group at the para position, the $S_{N} A r$ reaction tends to occur at the ortho position, which is consistent with classical $S_{N} A r$ reactivity. Notably, the overall yield for the three-step linear synthesis was $74 \%$. The programmed selective $\mathrm{C}-\mathrm{F}$ bond activation can also be extended to other perfluoroarenes such as polyfluorophenyl oxazoline $\mathbf{8}$. The oxazoline group in $\mathbf{8}$ serves as a directing group to facilitate the ortho $\mathrm{C}-\mathrm{F}$ bond activation via a palladium-catalyzed Suzuki-Miyaura coupling. ${ }^{90}$ Subsequent defluorosulfurization by our $S_{N} A r$ reaction delivered 10 in $55 \%$ yield. Lastly, we also examined iminedirected nickel-catalyzed double C-F bond functionalizations (Scheme $5 \mathrm{c}$ ), yielding product $11 .{ }^{91}$ To our delight, 11, despite its electron-donating aromatic substituents, participated in our $S_{N} A r$ reaction 
selectively at the position para to the aldehyde group and difluoroaryl product $\mathbf{1 2}$ was accessed in $85 \%$ yield.
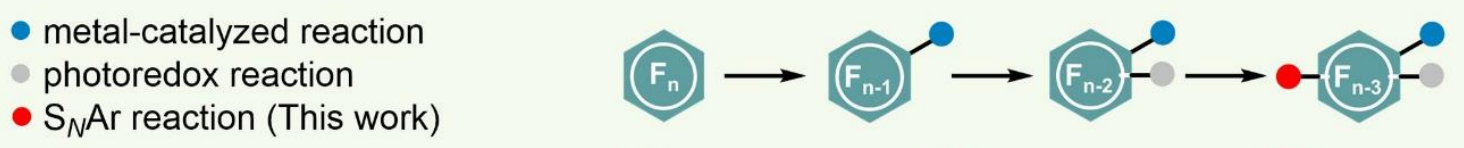
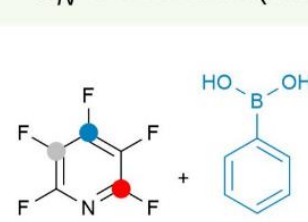<smiles>Fc1c(F)c(F)c(C2=NCCO2)c(F)c1F</smiles>

8

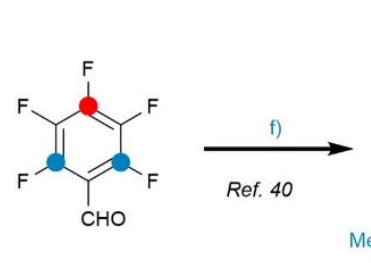

1 ae

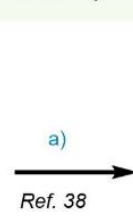

Ref. 38

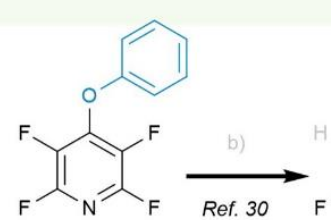

5

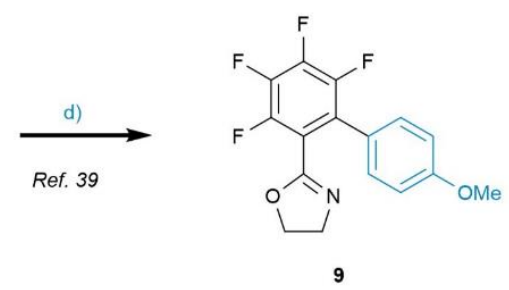

9

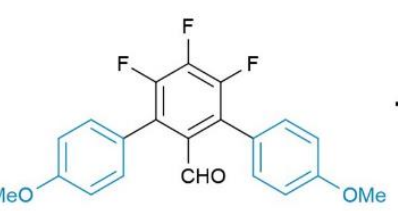

11

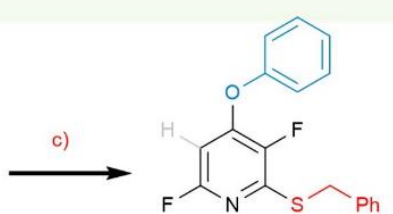

(a)

6

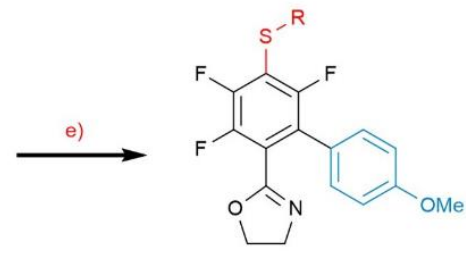

10

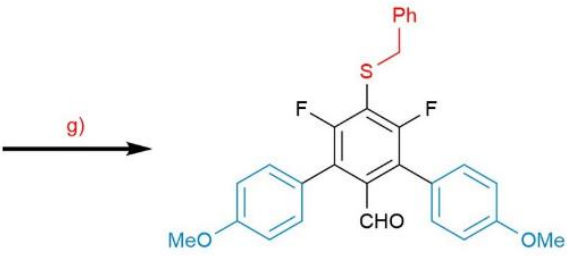

12

\section{Scheme 5. Programmed selective C-F bond activation of perfluoroarenes}

Reaction conditions:

${ }^{a}$ 1a $(5 \mathrm{mmol})$, phenylboronic acid $(10 \mathrm{mmol}), \mathrm{Ni}(\mathrm{acac})_{2}(0.25 \mathrm{mmol}), \mathrm{K}_{3} \mathrm{PO}_{4}(10 \mathrm{mmol}), \mathrm{THF}, \mathrm{O}_{2}$ balloon, reflux, $12 \mathrm{~h}, 87 \%$; ${ }^{\mathrm{b}} \mathbf{5}(0.2$ mmol), $\operatorname{lr}(\mathrm{ppy})_{3}$ (0.001 mmol), DIPEA (2 mmol), MeCN, Blue LEDs (456 nm), RT, $24 \mathrm{~h}, 99 \%$; 6 (0.1 mmol), benzyl mercaptan (0.1 $\mathrm{mmol}$ ), triethylamine (0.15 mmol), $\mathrm{MeCN}, \mathrm{N}_{2}, \mathrm{RT}, 12 \mathrm{~h}, 85 \%$; $8 \mathbf{8}(0.2 \mathrm{mmol})$, (4-methoxyphenyl)boronic acid (0.24 mmol), $\mathrm{Pd}(\mathrm{MeCN}) \mathrm{Cl}_{2}(0.020 \mathrm{mmol}), \operatorname{DPPF}(0.020 \mathrm{mmol}), \mathrm{Cs}_{2} \mathrm{CO}_{3}(0.4 \mathrm{mmol})$, toluene, $130{ }^{\circ} \mathrm{C}, 3 \mathrm{~h}, 87 \%$; 9 (0.1 mmol), benzyl mercaptan (0.1 mmol), triethylamine (0.15 mmol), $\mathrm{MeCN}, \mathrm{N}_{2}, 60^{\circ} \mathrm{C}, 12 \mathrm{~h}, 55 \%$; f $1 \mathrm{ae}(0.5 \mathrm{mmol}), \mathrm{BnNH}_{2}(0.5 \mathrm{mmol}), \mathrm{MgSO}_{4}(3.5 \mathrm{mmol})$, $\mathrm{DCM}, \mathrm{RT}, 1$ day, in one pot condition add (4-methoxyphenyl)boronic acid (1.5 mmol), Ni(cod) 2 (0.05 mmol), $\mathrm{PPh}_{3}(0.1 \mathrm{mmol})$, $\mathrm{K}_{2} \mathrm{CO}_{3}(1.5 \mathrm{mmol}), \mathrm{THF}, \mathrm{N}_{2}, 65^{\circ} \mathrm{C}, 48 \mathrm{~h}$, then $3 \mathrm{M} \mathrm{HCl}(\mathrm{aq}), \mathrm{RT}, 30 \mathrm{~min}, 20 \%$ for three steps; 811 (0.05 mmol), benzyl mercaptan (0.075 mmol), triethylamine $(0.125 \mathrm{mmol}), \mathrm{MeCN}, 60^{\circ} \mathrm{C}, 24 \mathrm{~h}, 85 \%$.

To further demonstrate the practicality of this $S_{N} A r$ reaction, we attempted to perform the reaction under mechanochemical and solvent-free conditions. ${ }^{92-93}$ Here, 2-mercaptobenzothiazole 2ab, 2,3,4,5,6pentafluorobenzaldehyde (1ae), and a base are introduced into a milling jar and ground at $30 \mathrm{~Hz}$ with $\mathrm{ZrO}_{2}$ milling balls (Table 2). Compared to the reaction in the solvent phase, the ball milling process provided a higher reaction rate ( $97 \%$ yield in $10 \mathrm{~min}$ vs. $90 \%$ yield in $1 \mathrm{~h}$ ). 
Table 2. Mechanochemical $S_{N} A r$ defluorosulfurization in a ball mill setup

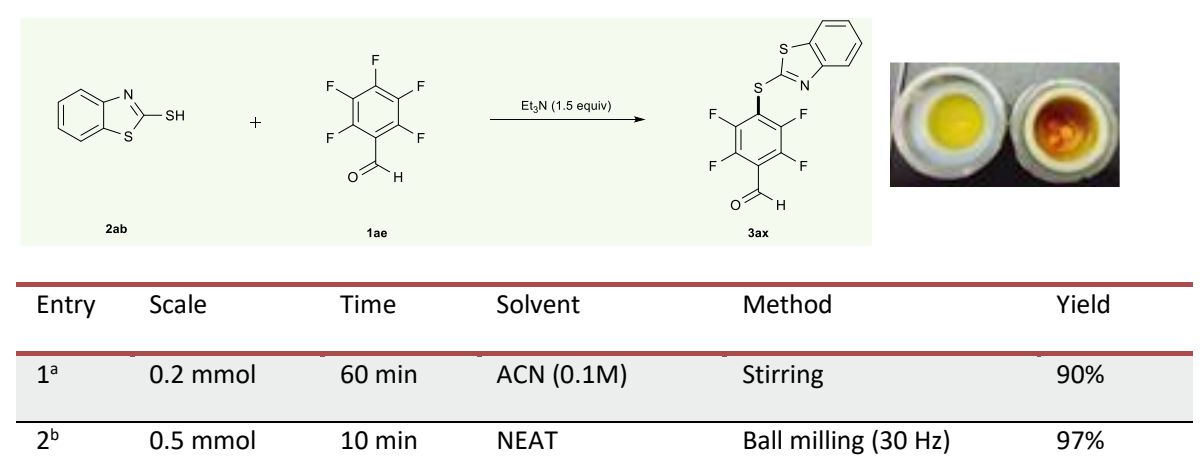

aReaction in batch up setup. 2ab $(0.2 \mathrm{mmol})$, 1ae $(0.2 \mathrm{mmol})$, triethylamine $(0.3 \mathrm{mmol})$, acetonitrile $(2.0 \mathrm{~mL}), 60 \mathrm{~min}$. ${ }^{\mathrm{b}} \mathrm{Reaction}$ in a ball milling setup. $2 \mathrm{ab}(0.5 \mathrm{mmol})$, $1 \mathrm{ae}(0.5 \mathrm{mmol})$, triethylamine $(0.75 \mathrm{mmol})$, ball milling, $30 \mathrm{~Hz}, 10 \mathrm{~min}$. See the Supporting Information for full experimental details.

\section{Nickel-catalyzed desulfurative arylation}

Recently, considerable efforts have been devoted to developing radical pathways to replace traditional reaction processes based on transition metals. To further demonstrate the synthetic utility of our newly developed $S_{N} A r$ method, we envisioned that the defluorosulfurinated products could act as electron acceptors in single-electron transfer processes. This could open up new opportunities for $\mathrm{C}\left(\mathrm{sp}^{3}\right)-\mathrm{S}$ bond activation that offer an alternative to the traditional strategy involving oxidative addition of the $\mathrm{C}-\mathrm{S}$ bond to a metal center. This is attractive as oxidative addition of mercaptan derivatives is often challenging owing to the strength of many $\mathrm{C}\left(\mathrm{sp}^{3}\right)-\mathrm{S}$ bonds. Hence, we considered an alternative strategy ${ }^{48-50}$ beginning with the conversion of aliphatic mercaptans into redox-active thioethers ${ }^{51-52}$ through our aforementioned $S_{N} A r$ protocol.

We anticipated that the LUMO of the perfluoroarene-containing thioether is lower in energy than alkyl mercaptans alone, which in turn makes them excellent single electron acceptors. ${ }^{94}$ Upon accepting an electron, the thioether becomes a transient radical anion that quickly undergoes heterolytic bond cleavage of its $\mathrm{C}\left(\mathrm{sp}^{3}\right)-\mathrm{S}$ bond to yield a perfluoroaryl thiolate anion and an alkyl radical (Scheme 2 ). With this concept, various perfluoroarylated substrates can be used to generate an alkyl radical upon undergoing single electron transfer. However, based on different arguments outlined in the SI (Section 7), e.g., cost, yield, and the electron-accepting character as quantified by the reduction potential of the thioether, 2,3,5,6-tetrafluoro-pyridine sulfide 3a seems to be the optimal substrate for this purpose. As the enhanced electron-accepting character of the thioether is expected to be more beneficial for the anticipated C-S activation process, we have also considered the possibility of adding $\mathrm{MgBr}_{2}$, a Lewis acid, to interact with the $N$ atom at the perfluoro-pyridinyl group to increase the propensity for its reduction. Cyclic voltammetry measurements show that the reduction potential of $3 a$ is $-1.26 \mathrm{~V}$ (vs. SCE in DMA, Fig S8.2.2e). However, in the presence of $\mathrm{MgBr}_{2}$, the reduction potential increases to $-1.20 \mathrm{~V}$, confirming that the addition of $\mathrm{MgBr}_{2}$ indeed does enhance the electron-accepting character of the thioether. ${ }^{19} \mathrm{~F}-\mathrm{NMR}$ measurements of $\mathbf{3 a}$ are also in line with this finding, as the presence of $\mathrm{MgBr}_{2}$ induces a 0.09 ppm low-field shift of 3a (Fig. S8.2.1).

Nickel-catalyzed desulfurative arylation - Optimization 
With these findings in hand, we explored the utility of perfluoro-thioether $\mathbf{3 a}$ as a substrate in the crosselectrophile reductive cross-coupling reaction with 4-bromoacetophenone (13a) (Table 3). We envisioned that nickel, with appropriate choice of ligand, could furnish the desired cross-coupling product 14a. Winnowing out incompetent reaction conditions, we discovered that $\mathrm{NiCl}_{2}(\mathrm{BCP})(10 \mathrm{~mol} \%)$ with $\mathrm{Mn}(0.2 \mathrm{mmol})$ and $\mathrm{MgBr}_{2}$ could provide 14a in $99 \% \mathrm{GC}$ yield after stirring at $60^{\circ} \mathrm{C}$ for 16 hours (Entry 1). Further purification of the crude mixture gave product 14a in a remarkable $97 \%$ isolated yield. Other nickel catalysts, such as $\mathrm{Ni}(\operatorname{cod})_{2}(83 \%$ yield, Entry 2$), \mathrm{NiCl}_{2} \cdot$ glyme ( $83 \%$ yield, Entry 3 ), and $\mathrm{NiCl}_{2}$ (dppp) (95\% yield, Entry 4) were also examined and delivered product 14a in relatively good, yet inferior yields. Similarly, other bipyridine ligands were screened; however, L2 (34\% yield, Entry 5) and L3 (68\% yield, Entry 6) showed a deleterious effect on the reaction yield. Interestingly, the absence of $\mathrm{MgBr}_{2}$ (88\% yield, Entry 7) and its substitution with $\mathrm{MgCl}_{2}$ (80\% yield, Entry 8 ) decreases the yield. In addition, replacing $\mathrm{Mn}$ with other metallic reducing agents, such as Zn powder (Entry 9) and Mg powder (Entry 10), resulted in no observable reaction. Lastly, using DMA in lieu of DMF resulted in a modest decrease in the reaction ( $90 \%$ yield, Entry 11 ).

Table 3. Optimization of nickel-catalyzed desulfurative arylations

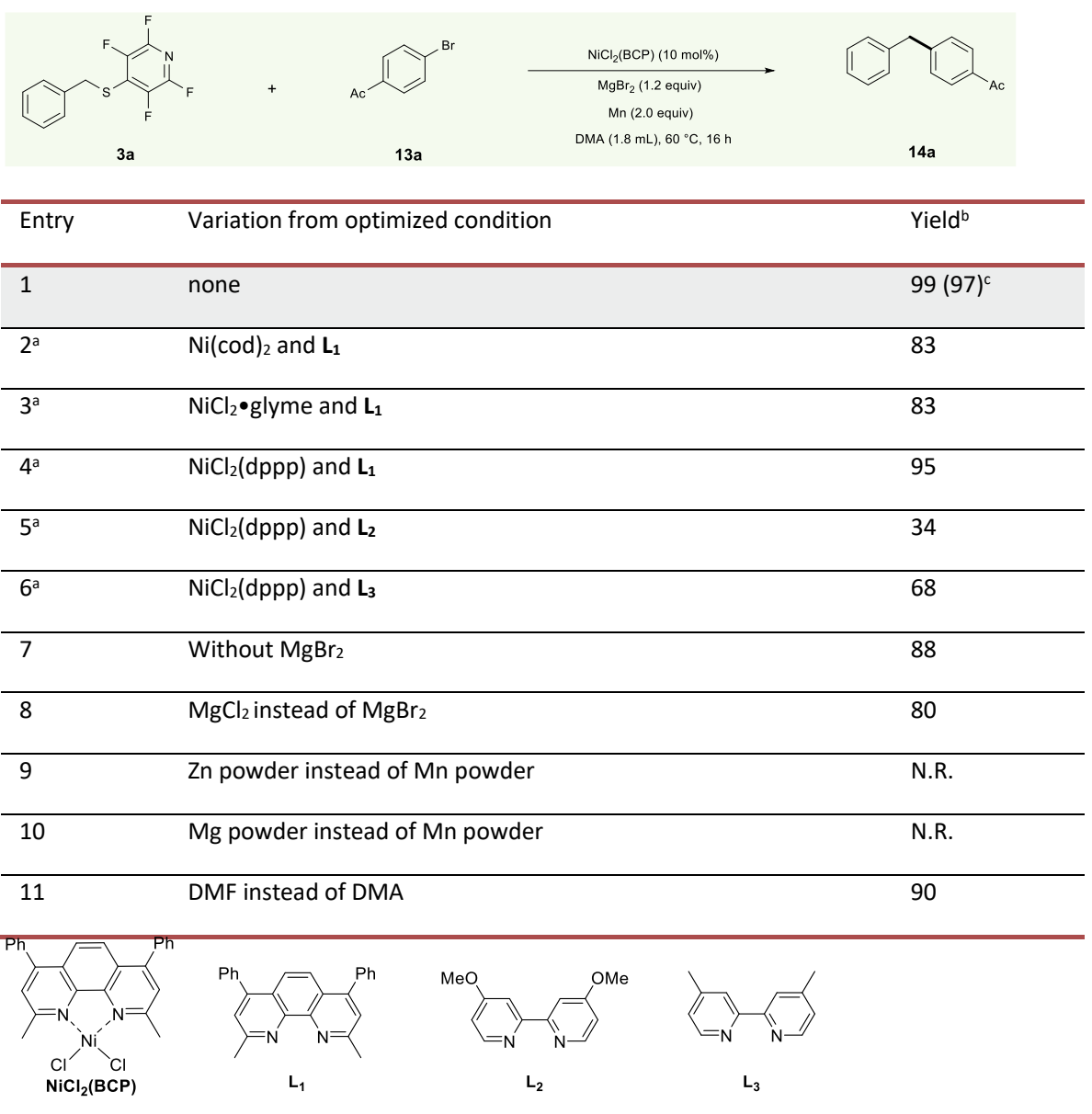

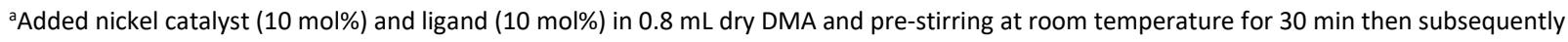
added 3a (0.1 mmol), 13a (0.1 mmol), $\mathrm{MgBr}_{2}(1.2 \mathrm{mmol}), \mathrm{Mn}(2.0 \mathrm{mmol})$ and $1.0 \mathrm{~mL}$ dry DMA. betermined by GC-MS of the crude reaction mixture using $n$-decane as internal standard. Clsolated yield after purification by column chromatography. See the Supporting Information for more entries in our optimization studies. 


\section{Nickel-catalyzed desulfurative arylation - Substrate scope}

After establishing the optimal reaction condition, we explored the scope of our nickel-catalyzed desulfurative arylation (Scheme 6). The functional group tolerance of the aryl halide partner was first examined. As expected, a wide range of carbonyl group such as ketone, aldehyde, ester, and thioester are all tolerated and delivered the corresponding products $14 \mathrm{a}-14 \mathrm{~g}$ in 70-97\% yield. Particularly, 14d and $14 \mathrm{e}$ with different substitution positions of the ester group merely presented minor differences in reactivity, even though the ester at the ortho position in 14d imposes steric hindrance in the oxidative addition step with the nickel catalyst. We also found that using $\mathbf{1 3 g}$, which has a thioester moiety, could be coupled effectivity. Additionally, benzonitrile $\mathbf{1 3 h}$ delivered the corresponding product in $92 \%$ (14h), and the trifluoromethyl-containing product $14 \mathbf{i}$ could be prepared in $55 \%$ yield. It is remarkable that pharmacophores, such as sulfone and sulfonamide, endured the optimal reaction condition and afforded the corresponding diarylmethanes (14j-14k) in good to excellent yields (67-80\%). Moreover, we examined several aromatic systems commonly found in pharmaceutical agents, including naphthalene (13I) and quinoline (13m-13n). These substrates afforded desulfurization products $\mathbf{1 4 l - 1 4 n}$ in moderate to good yields (55-80\%). The reaction conditions were also applied to trifluorinated pyridine 130, which afforded the desired product 140 in $65 \%$ yield.

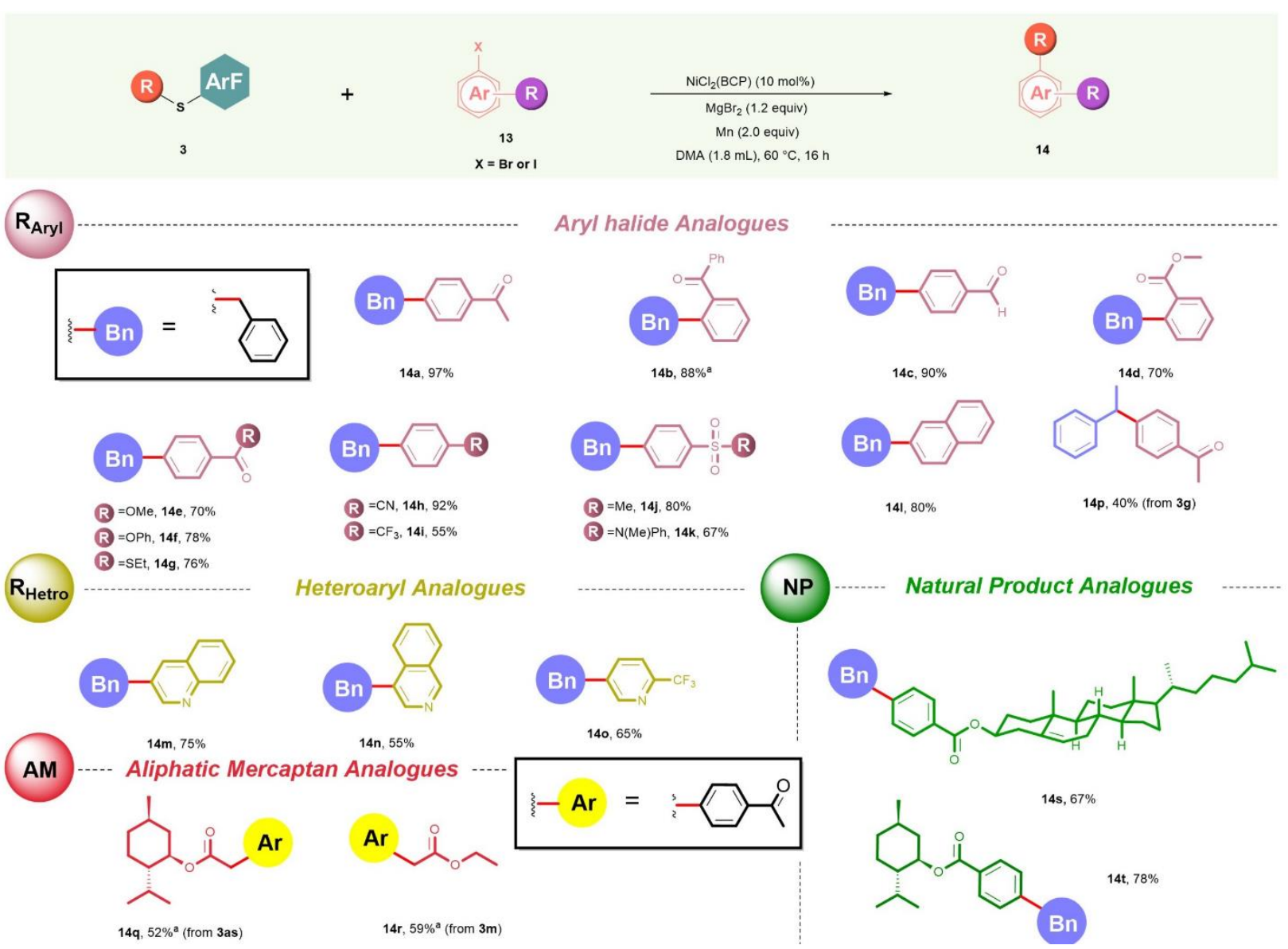

Scheme 6. Scope evaluation of nickel-catalyzed desulfurative arylation of aryl halide and mercaptans

Reaction conditions: 3 (0.1 mmol), 13a (0.12 mmol), NiCl $2(B C P)(10 \mathrm{~mol} \%), \mathrm{Mn}$ powder (0.2 mmol), $\mathrm{MgBr}_{2}(0.12 \mathrm{mmol}), \mathrm{dry}$ DMA (1.8 mL). aFrom aryl iodide. 
Next, we turned our attention to the investigation of the coupling reaction between 13a and different mercaptan analogues. Due to the stabilization of the radical generated from secondary benzylic mercaptan 3p, homocoupling side products were expected, which contributed to the lower yield (14p, $40 \%$ ) compared to the primary system $3 a(14 a, 97 \%$ - vide supra). If the benzene ring was replaced by either a bulky or small aliphatic ester scaffold (3as and $3 \mathrm{~m}$ ), nickel-catalyzed reductive cross-coupling was found to proceed and give reasonable yields (52-59\%). The superlative tolerance of various functionalities of this reductive cross-coupling scheme, especially for esters, has been demonstrated on the late-stage functionalization of natural products such as cholesterol (14s) and menthol (14t). As for further application, this nickel-mediated aryl C-S bond formation might provide a promising chemical approach for cholesterol-based drug carriers. ${ }^{95}$

\section{Nickel-catalyzed desulfurative arylation - Mechanistic studies}

A series of radical trapping experiments were performed to elucidate the underlying mechanism of our nickel-catalyzed desulfurative arylation (Table 4). Two major conclusions can be drawn from the results; first, the benzylic radical, derived from 13a, could be trapped under our standard conditions using TEMPO. This led to the formation of $\mathbf{1 5}$ in $12 \%$ NMR yield, while the cross-coupling product 14a was not detected (Entry 1). This confirms that the reaction is likely to progress via a radical pathway. Secondly, neither $\mathrm{Ni}^{\prime \prime}$ nor $\mathrm{Mn}^{0}$ alone can generate the benzyl radical (Entries 2-3). Hence, we hypothesized that $\mathrm{Ni}^{\prime \prime}$ is a precatalyst that is reduced in situ to generate a catalytic active nickel species. $\mathrm{Ni}^{\prime \prime}$ is reduced by $\mathrm{Mn}$ powder owing to the lower reduction potential of the latter, with a value of $-1.14 \mathrm{~V}$ versus SCE in DMA according to our own cyclic voltammetry experiments (Section 8.2.2 of the $\mathrm{SI}$ ). It is entirely feasible that

Table 4. Mechanism studies of nickel-catalyzed desulfurative arylation

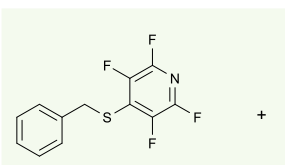

$3 a$

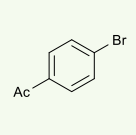

$13 a$

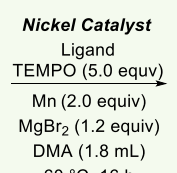

$60^{\circ} \mathrm{C}, 16 \mathrm{~h}$

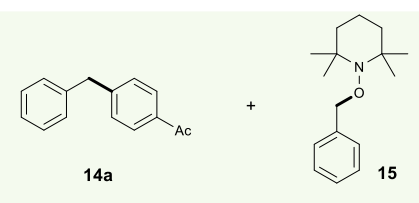

\begin{tabular}{llcc}
\hline Entry & TEMPO electron trapping experiment & $14 a^{\mathrm{b}}$ & $15^{\mathrm{c}}$ \\
\hline 1 & $\mathrm{NiCl}$ (BCP) (10 mol\%) & $0 \%$ & $12 \%$ \\
2 & $\mathrm{NiCl}$ (BCP) (10 mol\%) without Mn powder & $0 \%$ & $0 \%$ \\
3 & Without nickel catalyst and ligand & $0 \%$ & $0 \%$ \\
\hline
\end{tabular}

Additional confirmatory experiment

\begin{tabular}{llcc}
\hline $4^{\mathrm{a}}$ & $\mathrm{Ni}(\operatorname{cod}) 2(10 \mathrm{~mol} \%), \mathrm{L} 1(10 \mathrm{~mol} \%)$ & $0 \%$ & $13 \%$ \\
$5^{\mathrm{a}}$ & $\mathrm{Ni}(\operatorname{cod}) 2(10 \mathrm{~mol} \%), \mathrm{L} 1(10 \mathrm{~mol} \%)$ without 13a & $0 \%$ & $26 \%$ \\
$6^{\mathrm{a}}$ & $\mathrm{Ni}(\operatorname{cod}) 2(10 \mathrm{~mol} \%), \mathrm{L} 1(10 \mathrm{~mol} \%)$ without TEMPO & $67 \%$ & $0 \%$
\end{tabular}

Reaction conditions: $3 a(0.1 \mathrm{mmol}), 13 a(0.12 \mathrm{mmol}), \mathrm{NiCl}_{2}(\mathrm{BCP})$ (10 mol\%), $\mathrm{Mn}$ powder (0.2 mmol), $\mathrm{MgBr} 2$ (0.12 mmol) and TEMPO (0.5 mmol) in dry DMA (1.8 mL). added nickel catalyst (10 mol\%) and ligand (10 mol\%) in 0.8mL DMA and pre-stirring at room temperature for $30 \mathrm{~min}$ then subsequently added $3 a(0.1 \mathrm{mmol}), 13 a(0.1 \mathrm{mmol}), \mathrm{MgBr}_{2}$ (1.2 mmol), $\mathrm{Mn}(2.0 \mathrm{mmol})$ and TEMPO $(0.5 \mathrm{mmol})$ in dry DMA $(1.0 \mathrm{~mL})$. b Determined by GC-MS of the crude reaction mixture using $n$-decane as internal standard. 'Determined by NMR of the crude reaction mixture using tetrachloroethane as internal standard. 
the mechanism begins from a $\mathrm{Ni}^{0}$ oxidation state (Table 3, Entry 2). Moreover, we employed $\mathrm{Ni}(\mathrm{cod})_{2}$ as a model for the active catalyst in the absence of 13a and detected 15 in $26 \%$ yield from our GCMS assay (Entry 4). However, adding 13a to the reaction reduced the yield of 15 to $13 \%$ (Entry 5). This observation alludes that $\mathrm{Ni}^{0}$ behaves as a bifunctional catalyst that undergoes oxidative addition with 13a in parallel to the single-electron transfer process to generate the benzyl radical.

To confirm this, we performed the reaction with a stoichiometric amount of $\mathrm{Ni}^{0}$ species but no $\mathrm{Mn}$ powder and TEMPO (Entry 6). Under the assumption that only $\mathrm{Ni}^{\circ}$ can contribute to the radical formation and the oxidative addition step, the maximum yield for the cross-coupling product 14a should only be at $50 \% .{ }^{96}$ Interestingly, we observed in Entry 6 that 14 a was obtained in $67 \%$ yield, which clearly exceeds $50 \%$. This suggests that other $\mathrm{Ni}$ species like $\mathrm{Ni}^{\prime}$ might also influence the reaction. In other words, $\mathrm{Ni}^{1}$ might also participate in producing alkyl radicals and in the oxidative addition with 13a as a coexisting alternative pathway.

Based on the preliminary experimental evidence gathered, we propose the mechanism outlined in Figure 3. But for simplicity, we only illustrated the catalytic cycle focusing on $\mathrm{Ni}^{0}$ as the active catalyst. With the in situ formed active catalysts, $\mathrm{Ni}^{0} \mathrm{I}$, the generation of the alkyl radical from $\mathbf{3}$ as well as the oxidative addition with $\mathbf{1 3}$ can occur in parallel. Insertion of the alkyl radical then generates $\mathrm{Ni}^{\text {III }}$ complex IV that undergoes reductive elimination to the $\mathrm{Ni}^{1}$ species $\mathbf{V}$, forging the desired $\mathrm{C}\left(\mathrm{sp}^{3}\right)-\mathrm{C}\left(\mathrm{sp}^{2}\right)$ bond and delivering the cross-coupled product 14 . Finally, the active nickel catalyst is regenerated via a single electron transfer from $\mathrm{Mn}^{0}$ to $\mathrm{Ni}^{1} .^{97}$

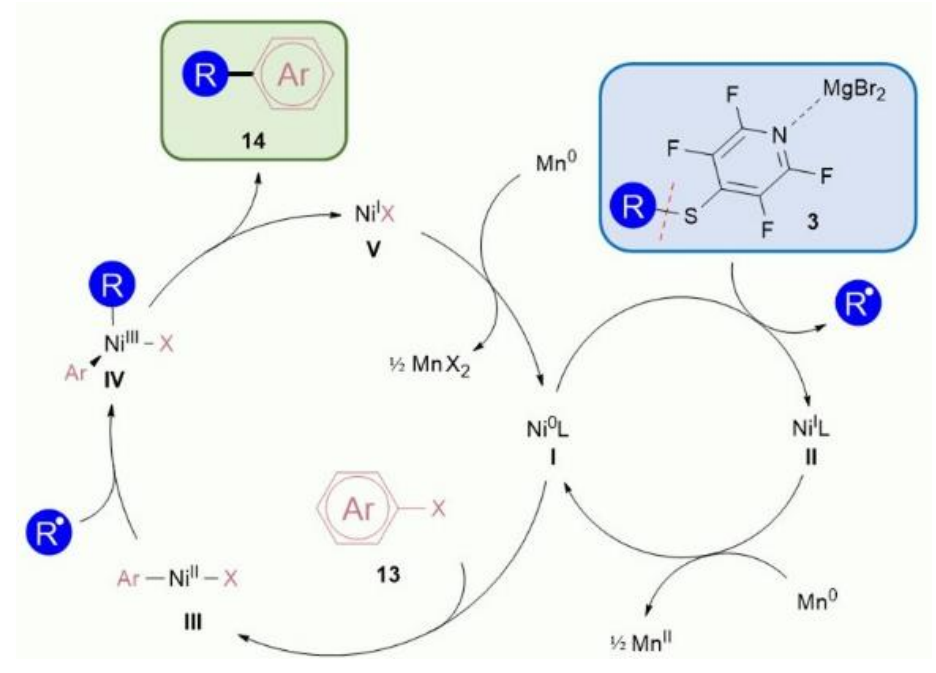

Figure 3. Proposed mechanism of nickel-catalyzed desulfurative arylation

\section{Summary and Conclusions}

In conclusion, we have developed a catalyst-free, operationally simple defluorosulfurization protocol for per-/polyfluoroaromatic systems via a nucleophilic aromatic substitution $\left(\mathrm{S}_{N} \mathrm{Ar}\right)$ step. The mild condition allows a wide range of readily available alkyl- and aryl-mercaptans as well as natural product derivatives containing thiol groups to be efficiently converted into valuable per-/polyfluoroaryl sulfides with nearcomplete regioselectivity. With the help of quantum chemical calculations, we have been able to 
address some questions about the mechanism of this interesting $S_{N} A r$ reaction. We could rationalize why only one TS could be located for several $S_{N} A r$ reactions with perfluoroaromatics, typically in electron-poor systems for which a stepwise mechanism is expected. A key to understanding this behavior is to use a Marcus-type picture and to interpret the studied reactions as two-step processes, consisting of an initial "normal" step and a second barrierless step. The value of our newly developed strategy to yield defluorosulfurinated compounds was demonstrated by using the products as partners in nickel-catalyzed reductive desulfurization reactions. The combination of this process with the aforementioned $S_{N} A r$ step has proven to be a powerful tool that enables the transformation of native thiol groups into a diverse range of arenes. In the context of the desulfurization step, we have been able to provide some new insights into the mechanistic details. We demonstrate that the breakage of the $C$

- S bond proceeds via a radical transfer event and showed that the Ni catalyst concurrently reduces the perfluoroaryl-containing thioether to generate an alkyl radical and undergoes oxidative addition with the aryl halides.

\section{ACKNOWLEDGMENTS}

The authors thank Prof. Ching Tat To (THU) for the instructions on the use of the ball milling equipment. We further like to thank Prof. Chi Wi Ong (NSYSU) for sharing laboratory space and reagents. We also thank Prof. Vincent Wang (NSYSU) for discussions on the mechanisms. Y.-T. T. acknowledges support from the research project for undergraduate students of NSYSU. The authors thank the National Center for High-performance Computing (NCHC) for providing computational and storage resources. This work was supported by the Young Scholar Fellowship Program by the Ministry of Science and Technology in Taiwan (MOST 109-2636-M-110-005 and MOST 109-2636-M-110-004) and Yushan Young Scholar Program (Ministry of Education, Taiwan)

\section{DECLARATION OF INTERESTS}

The authors declare no competing interests.

\section{REFERENCES}

1. Terrier, F. (2013). Modern nucleophilic aromatic substitution (John Wiley \& Sons).

2. Bunnett, J.F., and Zahler, R.E. (1951). Aromatic Nucleophilic Substitution Reactions. Chem. Rev. 49, 273-412.

3. Mortier, J. (2015). Arene chemistry: reaction mechanisms and methods for aromatic compounds (John Wiley \& Sons).

4. Boström, J., Brown, D.G., Young, R.J., and Keserü, G.M. (2018). Expanding the medicinal chemistry synthetic toolbox. Nat. Rev. Drug Discov. 17, 709-727.

5. Brown, D.G., and Bostrom, J. (2016). Analysis of past and present synthetic methodologies on medicinal chemistry: where have all the new reactions gone? Miniperspective. J. Med. Chem. $59,4443-4458$. 
6. Roughley, S.D., and Jordan, A.M. (2011). The medicinal chemist's toolbox: an analysis of reactions used in the pursuit of drug candidates. J. Med. Chem. 54, 3451-3479.

7. See, Y.Y., Morales-Colón, M.T., Bland, D.C., and Sanford, M.S. (2020). Development of $S_{N} A r$ Nucleophilic Fluorination: A Fruitful Academia-Industry Collaboration. Acc. Chem. Res. 53, 23722383.

8. Lennox, A.J.J. (2018). Meisenheimer complexes in $\mathrm{S}_{N} A r$ reactions: intermediates or transition states? Angew. Chemie Int. Ed. 57, 14686-14688.

9. Rohrbach, S., Smith, A.J., Pang, J.H., Poole, D.L., Tuttle, T., Chiba, S., and Murphy, J.A. (2019). Concerted nucleophilic aromatic substitution reactions. Angew. Chemie Int. Ed. 58, 1636816388.

10. You, Z., Higashida, K., Iwai, T., and Sawamura, M. (2021). Phosphinylation of Non-activated Aryl Fluorides through Nucleophilic Aromatic Substitution at the Boundary of Concerted and Stepwise Mechanisms. Angew. Chemie Int. Ed. 60, 5778-5782.

11. Mallick, S., Xu, P., Würthwein, E., and Studer, A. (2019). Silyldefluorination of fluoroarenes by concerted nucleophilic aromatic substitution. Angew. Chemie Int. Ed. 58, 283-287.

12. Neumann, C.N., Hooker, J.M., and Ritter, T. (2016). Concerted nucleophilic aromatic substitution with ${ }^{19} \mathrm{~F}$ - and ${ }^{18} \mathrm{~F}-$. Nature 534, 369-373.

13. Kvíčala, J., Beneš, M., Paleta, O., and Král, V. (2010). Regiospecific nucleophilic substitution in 2, 3, 4, 5, 6-pentafluorobiphenyl as model compound for supramolecular systems. Theoretical study of transition states and energy profiles, evidence for tetrahedral SN2 mechanism. J. Fluor. Chem. 131, 1327-1337.

14. Singh, H. (2020). A DFT investigation on aromatic nucleophilic substitution ( $\left.\mathrm{S}_{N} \mathrm{Ar}\right)$ reaction between 4-fluoro-1-naphthaldehyde/4-fluoro-2-naphthaldehyde/1-fluoro-2-naphthaldehyde/1fluoronaphthalene and methylthiolate ion in gas phase and in protic/aprotic solvents. Struct. Chem. 31, 2205-2213.

15. Baker, J., and Muir, M. (2010). The Meisenheimer model for predicting the principal site for nucleophilic substitution in aromatic perfluorocarbons-Generalization to include ring-nitrogen atoms and non-fluorine ring substituents. Can. J. Chem. 88, 588-597.

16. Kim, C.-Y., Chang, J.S., Doyon, J.B., Baird, T.T., Fierke, C.A., Jain, A., and Christianson, D.W. (2000). Contribution of fluorine to protein- ligand affinity in the binding of fluoroaromatic inhibitors to carbonic anhydrase II. J. Am. Chem. Soc. 122, 12125-12134.

17. Scozzafava, A., Menabuoni, L., Mincione, F., Briganti, F., Mincione, G., and Supuran, C.T. (2000). Carbonic anhydrase inhibitors: perfluoroalkyl/aryl-substituted derivatives of aromatic/heterocyclic sulfonamides as topical intraocular pressure-lowering agents with prolonged duration of action. J. Med. Chem. 43, 4542-4551.

18. Sakamoto, Y., Suzuki, T., Kobayashi, M., Gao, Y., Fukai, Y., Inoue, Y., Sato, F., and Tokito, S. (2004). Perfluoropentacene: High-Performance $p-n$ junctions and complementary circuits with pentacene. J. Am. Chem. Soc. 126, 8138-8140. 
19. Murphy, A.R., and Frechet, J.M.J. (2007). Organic semiconducting oligomers for use in thin film transistors. Chem. Rev. 107, 1066-1096.

20. Sakamoto, Y., Suzuki, T., Miura, A., Fujikawa, H., Tokito, S., and Taga, Y. (2000). Synthesis, characterization, and electron-transport property of perfluorinated phenylene dendrimers. J. Am. Chem. Soc. 122, 1832-1833.

21. Müller, K., Faeh, C., and Diederich, F. (2007). Fluorine in pharmaceuticals: looking beyond intuition. Science 317, 1881-1886.

22. Wang, J., Sánchez-Roselló, M., Aceña, J.L., Del Pozo, C., Sorochinsky, A.E., Fustero, S., Soloshonok, V.A., and Liu, H. (2014). Fluorine in pharmaceutical industry: fluorine-containing drugs introduced to the market in the last decade (2001-2011). Chem. Rev. 114, 2432-2506.

23. Caron, S. (2020). Where does the fluorine come from? A review on the challenges associated with the synthesis of organofluorine compounds. Org. Process Res. Dev. 24, 470-480.

24. Szpera, R., Moseley, D.F.J., Smith, L.B., Sterling, A.J., and Gouverneur, V. (2019). The fluorination of $\mathrm{C}-\mathrm{H}$ bonds: developments and perspectives. Angew. Chemie Int. Ed. 58, 14824-14848.

25. Neumann, C.N., and Ritter, T. (2015). Late-stage fluorination: fancy novelty or useful tool? Angew. Chemie Int. Ed. 54, 3216-3221.

26. Amii, H., and Uneyama, K. (2009). C- F bond activation in organic synthesis. Chem. Rev. 109, 2119-2183.

27. Ahrens, T., Kohlmann, J., Ahrens, M., and Braun, T. (2015). Functionalization of fluorinated molecules by transition-metal-mediated $\mathrm{C}-\mathrm{F}$ bond activation to access fluorinated building blocks. Chem. Rev. 115, 931-972.

28. Weaver, J., and Senaweera, S. (2014). C-F activation and functionalization of perfluoro-and polyfluoroarenes. Tetrahedron 41, 7413-7428.

29. Senaweera, S., and Weaver, J.D. (2016). Photocatalytic C-F Reduction and Functionalization. Aldrichimica Acta 49, 45.

30. Kuehnel, M.F., Lentz, D., and Braun, T. (2013). Synthesis of fluorinated building blocks by transition-metal-mediated hydrodefluorination reactions. Angew. Chemie Int. Ed. 52, 33283348.

31. Niwa, T., Ochiai, H., Watanabe, Y., and Hosoya, T. (2015). Ni/Cu-catalyzed defluoroborylation of fluoroarenes for diverse C-F bond functionalizations. J. Am. Chem. Soc. 137, 14313-14318.

32. Do, H.-Q., and Daugulis, O. (2008). Copper-catalyzed arylation and alkenylation of polyfluoroarene C- H bonds. J. Am. Chem. Soc. 130, 1128-1129.

33. Arora, A., and Weaver, J.D. (2016). Visible Light Photocatalysis for the generation and use of reactive azolyl and polyfluoroaryl intermediates. Acc. Chem. Res. 49, 2273-2283. 
34. Pistritto, V.A., Schutzbach-Horton, M.E., and Nicewicz, D.A. (2020). Nucleophilic aromatic substitution of unactivated fluoroarenes enabled by organic photoredox catalysis. J. Am. Chem. Soc. $142,17187-17194$.

35. Abernethy, D.R., DeStefano, A.J., Cecil, T.L., Zaidi, K., and Williams, R.L. (2010). Metal impurities in food and drugs. Pharm. Res. 27, 750-755.

36. Balaram, V. (2016). Recent advances in the determination of elemental impurities in pharmaceuticals-Status, challenges and moving frontiers. Trac Trends Anal. Chem. 80, 83-95.

37. Feng, M., Tang, B., H Liang, S., and Jiang, X. (2016). Sulfur containing scaffolds in drugs: synthesis and application in medicinal chemistry. Curr. Top. Med. Chem. 16, 1200-1216.

38. Ling, T., Tran, M., González, M.A., Gautam, L.N., Connelly, M., Wood, R.K., Fatima, I., MirandaCarboni, G., and Rivas, F. (2015). (+)-Dehydroabietylamine derivatives target triple-negative breast cancer. Eur. J. Med. Chem. 102, 9-13.

39. Sittihan, S., Jumpathong, W., Sopha, P., and Ruchirawat, S. (2020). Synthesis and antitumor activity of bis (arylsulfonyl) dihydroimidazolinone derivatives. Bioorg. Med. Chem. Lett. 30, 126776.

40. Porter, D.W., Bradley, M., Brown, Z., Canova, R., Charlton, S., Cox, B., Hunt, P., Kolarik, D., Lewis, S., and O'Connor, D. (2014). The discovery of potent, orally bioavailable pyrazolo and triazolopyrimidine CXCR2 receptor antagonists. Bioorg. Med. Chem. Lett. 24, 72-76.

41. Arisawa, M., Ichikawa, T., and Yamaguchi, M. (2012). Rhodium-catalyzed synthesis of diaryl sulfides using aryl fluorides and sulfur/organopolysulfides. Org. Lett. 14, 5318-5321.

42. Arisawa, M., Suzuki, T., Ishikawa, T., and Yamaguchi, M. (2008). Rhodium-catalyzed substitution reaction of aryl fluorides with disulfides: $p$-orientation in the polyarylthiolation of polyfluorobenzenes. J. Am. Chem. Soc. 130, 12214-12215.

43. Mulryan, D., White, A.J.P., and Crimmin, M.R. (2020). Organocatalyzed Fluoride Metathesis. Org. Lett. 22, 9351-9355.

44. Schmidt, A., Mordhorst, T., Namyslo, J.C., and Telle, W. (2007). Hetarenium salts from pentafluoropyridine. Syntheses, spectroscopic properties, and applications. J. Heterocycl. Chem. 44, 679-684.

45. Park, N. H., dos Passos Gomes, G., Fevre, M., Jones, G. O., Alabugin, I. V., \& Hedrick, J. L. (2017). Organocatalyzed synthesis of fluorinated poly (aryl thioethers). Nat. Commun. 8, 166.

46. Spokoyny, A.M., Zou, Y., Ling, J.J., Yu, H., Lin, Y.-S., and Pentelute, B.L. (2013). A perfluoroarylcysteine SNAr chemistry approach to unprotected peptide stapling. J. Am. Chem. Soc. 135, 5946-5949.

47. Fadzen, C.M., Wolfe, J.M., Zhou, W., Cho, C.-F., von Spreckelsen, N., Hutchinson, K.T., Lee, Y.-C., Chiocca, E.A., Lawler, S.E., and Yilmaz, O.H. (2020). A Platinum (IV) Prodrug-Perfluoroaryl Macrocyclic Peptide Conjugate Enhances Platinum Uptake in the Brain. J. Med. Chem. 63, 67416747. 
48. The idea to utilize the perfluoroarene as a redox-active scaffold was conceptualized through references 48-50. Smith, M., March, J. March's advanced organic chemistry: reactions, mechanisms, and structure. 6th ed. New Jersey: John Wiley \& Sons; 2006. Chapter 14, Substitution reactions: free radicals; p. 863-4

49. Marquet, J., Casado, F., Cervera, M., Espin, M., Gallardo, I., Mir, M., and Niat, M. (1995). Reductively activated'polar'nucleophilic aromatic substitution. A new mechanism in aromatic chemistry? Pure Appl. Chem. 67, 703-710.

50. Marquet, J., Jiang, Z., Gallardo, I., Batlle, A., and Cayón, E. (1993). Reductively activated "polar" nucleophilic aromatic substitution of pentafluoronitrobenzene. The SRN2 hypothesis revisited. Tetrahedron Lett. 34, 2801-2804.

51. Upon the completion of the project and during the manuscript preparation, the Dilman group published a similar radical reaction. However, their strategy is to activate the $\mathrm{C}-\mathrm{H}$ bonds in unactivated alkanes through thiolation with S-tetrafluoropyridine disulfide to form a new C-S bond, which serves as a redox-active precursor for radical generation. Alternatively, our strategy mainly uses perfluoroarenes to activate native thiol compounds for desulfurization reaction. See reference 51 and 52. Panferova, L.I., Zubkov, M.O., Kokorekin, V.A., Levin, V. V, and Dilman, A.D. (2021). Using the Thiyl Radical for Aliphatic Hydrogen-Atom Transfer: Thiolation of Unactivated C- H Bonds. Angew. Chemie Int. Ed. 60, 2849-2854.

52. Panferova, L.I., and Dilman, A.D. (2021). Light-Mediated Sulfur-Boron Exchange. Org. Lett. 23, 3919-3922.

53. Song, C., and Ma, X. (2003). New design approaches to ultra-clean diesel fuels by deep desulfurization and deep dearomatization. Appl. Catal. B Environ. 41, 207-238.

54. Stanislaus, A., Marafi, A., and Rana, M.S. (2010). Recent advances in the science and technology of ultra low sulfur diesel (ULSD) production. Catal. Today 153, 1-68.

55. Weix, D.J. (2015). Methods and mechanisms for cross-electrophile coupling of Csp2 halides with alkyl electrophiles. Acc. Chem. Res. 48, 1767-1775.

56. Knappke, C.E.I., Grupe, S., Gärtner, D., Corpet, M., Gosmini, C., and Jacobi von Wangelin, A. (2014). Reductive cross-coupling reactions between two electrophiles. Chem. Eur. J. 20, 68286842.

57. Everson, D.A., and Weix, D.J. (2014). Cross-electrophile coupling: principles of reactivity and selectivity. J. Org. Chem. 79, 4793-4798.

58. Bougault, J., Cattelain, E., and Chabrier, P. (1940). New Method for Obtaining Benzene and Toluene Free of Thiophene and Methylthiophene. Bull. Soc. Chim. Fr 7, 781.

59. Pettit, G.R., and van Tamelen, E.E. (2004). Desulfurization with Raney Nickel. Org. React. 12, 356-529.

60. Li, J., Chen, J., Sang, R., Ham, W.-S., Plutschack, M.B., Berger, F., Chabbra, S., Schnegg, A., Genicot, C., and Ritter, T. (2020). Photoredox catalysis with aryl sulfonium salts enables siteselective late-stage fluorination. Nat. Chem. 12, 56-62. 
61. Berger, F., and Ritter, T. (2021). Site-Selective Late-Stage C-H Functionalization via Thianthrenium Salts. DOI: 10.1055/s-0040-1706034

62. Péter, Á., Perry, G.J.P., and Procter, D.J. (2020). Radical C- C Bond Formation using Sulfonium Salts and Light. Adv. Synth. Catal. 362, 2135-2142.

63. Berger, F., Plutschack, M.B., Riegger, J., Yu, W., Speicher, S., Ho, M., Frank, N., and Ritter, T. (2019). Site-selective and versatile aromatic $\mathrm{C}-\mathrm{H}$ functionalization by thianthrenation. Nature $567,223-228$.

64. Aukland, M.H., Šiaučiulis, M., West, A., Perry, G.J.P., and Procter, D.J. (2020). Metal-free photoredox-catalysed formal $\mathrm{C}-\mathrm{H} / \mathrm{C}-\mathrm{H}$ coupling of arenes enabled by interrupted Pummerer activation. Nat. Catal. 3, 163-169.

65. Xu, P., Zhao, D., Berger, F., Hamad, A., Rickmeier, J., Petzold, R., Kondratiuk, M., Bohdan, K., and Ritter, T. (2020). Site-Selective Late-Stage Aromatic [18F] Fluorination via Aryl Sulfonium Salts. Angew. Chemie Int. Ed. 59, 1956-1960.

66. Quiclet-Sire, B., and Zard, S.Z. (2006). The degenerative radical transfer of xanthates and related derivatives: an unusually powerful tool for the creation of carbon-carbon bonds. Top Curr. Chem. 264, 201-236.

67. Quiclet-Sire, B., and Zard, S.Z. (2006). Powerful Carbon? Carbon Bond Forming Reactions Based on a Novel Radical Exchange Process. Chem. Eur. J. 12, 6002-6016.

68. Mazzarella, D., Magagnano, G., Schweitzer-Chaput, B., and Melchiorre, P. (2019). Photochemical organocatalytic borylation of alkyl chlorides, bromides, and sulfonates. ACS Catal. 9, 5876-5880.

69. Schweitzer-Chaput, B., Horwitz, M.A., de Pedro Beato, E., and Melchiorre, P. (2019). Photochemical generation of radicals from alkyl electrophiles using a nucleophilic organic catalyst. Nat. Chem. 11, 129-135.

70. Zhang, L., Si, X., Yang, Y., Witzel, S., Sekine, K., Rudolph, M., Rominger, F., and Hashmi, A.S.K. (2019). Reductive C-C Coupling by Desulfurizing Gold-Catalyzed Photoreactions. ACS Catal. 9, 6118-6123.

71. Qin, Q., Wang, W., Zhang, C., Song, S., and Jiao, N. (2019). A metal-free desulfurizing radical reductive $\mathrm{C}-\mathrm{C}$ coupling of thiols and alkenes. Chem. Commun. 55, 10583-10586.

72. Shi, S., Qiu, W., Miao, P., Li, R., Lin, X., and Sun, Z. (2021). Three-component radical homo Mannich reaction. Nat. Commun. 12, 1006.

73. Terrett, J.A., Cuthbertson, J.D., Shurtleff, V.W., and MacMillan, D.W.C. (2015). Switching on elusive organometallic mechanisms with photoredox catalysis. Nature 524, 330-334.

74. Zheng, B., Tang, F., Luo, J., Schultz, J.W., Rath, N.P., and Mirica, L.M. (2014). Organometallic nickel (III) complexes relevant to cross-coupling and carbon-heteroatom bond formation reactions. J. Am. Chem. Soc. 136, 6499-6504.

75. Mondal, P., Pirovano, P., Das, A., Farquhar, E.R., and McDonald, A.R. (2018). Hydrogen Atom Transfer by a High-Valent Nickel-Chloride Complex. J. Am. Chem. Soc. 140, 1834-1841. 
76. Tasker, S.Z., Standley, E.A., and Jamison, T.F. (2014). Recent advances in homogeneous nickel catalysis. Nature 509, 299-309.

77. Ma, Y., Cammarata, J., and Cornella, J. (2019). Ni-Catalyzed reductive Liebeskind-Srogl alkylation of heterocycles. J. Am. Chem. Soc. 141, 1918-1922.

78. DMSO is used as the solvent for this entry since $\mathrm{Na}_{2} \mathrm{CO}_{3}$ is only relatively soluble in this polar aprotic solvent and is scarcely soluble to other solvents evaluated.

79. Prat, D., Pardigon, O., Flemming, H.-W., Letestu, S., Ducandas, V., Isnard, P., Guntrum, E., Senac, T., Ruisseau, S., and Cruciani, P. (2013). Sanofi's solvent selection guide: A step toward more sustainable processes. Org. Process Res. Dev. 17, 1517-1525.

80. Halama, A., Jirman, J., Boušková, O., Gibala, P., and Jarrah, K. (2010). Improved process for the preparation of montelukast: Development of an efficient synthesis, identification of critical impurities and degradants. Org. Process Res. Dev. 14, 425-431.

81. Senaweera, S.M., Singh, A., and Weaver, J.D. (2014). Photocatalytic hydrodefluorination: facile access to partially fluorinated aromatics. J. Am. Chem. Soc. 136, 3002-3005.

82. Bhambra, A.S., Edgar, M., Elsegood, M.R.J., Horsburgh, L., Kryštof, V., Lucas, P.D., Mojally, M., Teat, S.J., Warwick, T.G., and Weaver, G.W. (2016). Novel fluorinated benzimidazole-based scaffolds and their anticancer activity in vitro. J. Fluor. Chem. 188, 99-109.

83. Kwan, E.E., Zeng, Y., Besser, H.A., and Jacobsen, E.N. (2018). Concerted nucleophilic aromatic substitutions. Nat. Chem. 10, 917-923.

84. Rohrbach, S., Murphy, J.A., and Tuttle, T. (2020). Computational study on the boundary between the concerted and stepwise mechanism of bimolecular $\mathrm{S}_{N}$ Ar reactions. J. Am. Chem. Soc. 142, 14871-14876.

85. Zdilla, M.J., Dexheimer, J.L., and Abu-Omar, M.M. (2007). Hydrogen atom transfer reactions of imido manganese $(\mathrm{V})$ corrole: one reaction with two mechanistic pathways. J. Am. Chem. Soc. 129, 11505-11511.

86. Swansburg, S., Buncel, E., and Lemieux, R.P. (2000). Thermal racemization of substituted indolinobenzospiropyrans: Evidence of competing polar and nonpolar mechanisms. J. Am. Chem. Soc. 122, 6594-6600.

87. Ferrin, P., Simonetti, D., Kandoi, S., Kunkes, E., Dumesic, J.A., Nørskov, J.K., and Mavrikakis, M. (2009). Modeling ethanol decomposition on transition metals: a combined application of scaling and Brønsted- Evans- Polanyi relations. J. Am. Chem. Soc. 131, 5809-5815.

88. Grampp, G. (1993). The Marcus inverted region from theory to experiment. Angew. Chemie Int. Ed. English 32, 691-693.

89. Zhang, J., Wu, J., Xiong, Y., and Cao, S. (2012). Synthesis of unsymmetrical biaryl ethers through nickel-promoted coupling of polyfluoroarenes with arylboronic acids and oxygen. Chem. Commun. 48, 8553-8555. 
90. Yu, D., Shen, Q., and Lu, L. (2012). Selective palladium-catalyzed C-F activation/carbon-carbon bond formation of polyfluoroaryl oxazolines. J. Org. Chem. 77, 1798-1804.

91. Sun, A.D., and Love, J.A. (2011). Nickel-catalyzed selective defluorination to generate partially fluorinated biaryls. Org. Lett. 13, 2750-2753.

92. Porcheddu, A., Colacino, E., De Luca, L., and Delogu, F. (2020). Metal-mediated and metalcatalyzed reactions under mechanochemical conditions. ACS Catal. 10, 8344-8394.

93. Wang, G.-W. (2013). Mechanochemical organic synthesis. Chem. Soc. Rev. 42, 7668-7700.

94. Alkorta, I., Rozas, I., and Elguero, J. (1997). An attractive interaction between the $\pi$-cloud of C6F6 and electron-donor atoms. J. Org. Chem. 62, 4687-4691.

95. Ruwizhi, N., and Aderibigbe, B.A. (2020). The efficacy of cholesterol-based carriers in drug delivery. Molecules 25, 4330-4369.

96. Under the assumption that only $\mathrm{Ni}^{0}$ species can contribute to the benzyl radical formation and the oxidative-addition process, we have determined the maximum yield for the overall reaction. It was calculated from a simple notion that regardless of the distribution ratio of $\mathrm{Ni}^{0}$ for the oxidative addition and radical generation processes, the yield cannot exceed $50 \%$ since both processes would limit each other. For example, when $70 \%$ of $\mathrm{Ni}^{0}$ is used up for the oxidative addition and $30 \%$ for the benzyl radical preparation, the cross-coupling product can only be obtained with $30 \%$ yield. The same analogy can be applied vice versa. In any case, the yield is capped to $50 \%$, which corresponds to the situation when each $50 \%$ of $\mathrm{Ni}^{0}$ is consumed in the radical formation step and the oxidative addition step.

97. A similar reduction potential $\left(E_{P}=-1.17 \mathrm{~V}\right.$ vs. $\left.\mathrm{SCE}\right)$ of Nickel complex was measured by Doyle group as well, which indicate that $\mathrm{Ni}^{1}$ intermediate can generate $\mathrm{Ni}^{0}$ through $\mathrm{Mn}$ as reducing species ( $E^{\text {red }}=-1.35 \mathrm{~V}$ vs. SCE). See: Shields, B.J., and Doyle, A.G. (2016). Direct C (sp3)-H cross coupling enabled by catalytic generation of chlorine radicals. J. Am. Chem. Soc. 138, 1271912722 